American Journal of Biochemistry and Biotechnology 7 (2): 43-54, 2011

ISSN 1553-3468

(C) 2011 A.G.E. Amr et al., This open access article is distributed under a Creative Commons Attribution

(CC-BY) 3.0 license

\title{
Anticancer Activities of Some New Synthesized Thiazolo[3,2-a]Pyrido[4,3-d]Pyrimidine Derivatives
}

\author{
${ }^{1,2}$ Ashraf M. Mohamed, ${ }^{2,3}$ Abdel-Galil E. Amr, ${ }^{1}$ Musaed A. Alsharari, \\ ${ }^{1}$ Husam R.M. Al-Qalawi, ${ }^{4}$ Mosa O. Germoush and ${ }^{3}$ Mohamed A. Al-Omar \\ ${ }^{1}$ Department of Chemistry, College of Science, Al-Jouf University, Sakaka, Al-Jouf, Saudi Arabia \\ ${ }^{2}$ Department of Applied Organic, National Research Centre, Dokki, Cairo, Egypt \\ ${ }^{3}$ Department of Pharmaceutical Chemistry, College of Pharmacy, \\ King Saud University, Saudi Arabia \\ ${ }^{4}$ Department of Biology, College of Science, Al-Jouf University, \\ Sakaka, Al-Jouf, Saudi Arabia
}

\begin{abstract}
Problem statement: This study describes the synthesis and anticancer activities of a new series of thiazolo[3,2-a]pyrimidines derivatives (2-7) using 3,5-bisarylmethylene-1-methyl-4piperidone and 4-aryl-8-arylmethelene-6-methylpyrido[4,3-d]pyrimidine-2(1H)thiones as a starting materials. Approach: The antitumor activities of the newly synthesized compounds 4-7 were evaluated utilizing 60 different human tumor cell lines, representing leukemia, melanoma, lung, colon, brain, ovary, breast and prostate as well as kidney. Results: Some of the tested compounds exhibited better in vitro antitumor activities at low concentration $\left(\log 10 \mathrm{GI}_{50}=-4.7\right)$ against the used human tumor cell lines. Conclusion: From the obtained results, we can conclude that pyrimidine moieties fused to N-methylpipredine ring are essential for antitumor activities. In the present work, we can suggest that the anticancer activity is due to the presence of nitrogen heterocyclic rings and the presence of sulfur atom generally enhancing the activity.
\end{abstract}

Key words: Synthesis, reactions, pyrimidinethione, thiazolopyrimidine, anticancer activity, starting materials, nitrogen heterocyclic, pyrimidines derivatives, biological activity

\section{INTRODUCTION}

Cancer poses a serious human health problem despite much progress in understanding its biology and pharmacology. Consequently, the design of new lead structures employed as antitumor agents is one of the most urgent research areas in contemporary medicinal chemistry. During our ongoing studies aimed at the discovery of new heterocycles endowed with antitumour activity, we have reported on the synthesis and antitumor activities of a series of heterocyclic compounds (Hammam et al., 2003; 2005; Amr et al., 2006; Velusamy and Palaniappan, 2011; Abd El-Salam et al., 2010). Pyrimidine has gained considerable attention because of its diversity in biological activity and widespread applications in pharmaceuticals fields (Katritzky and Rees, 1996; Francis et al., 2011). For instance, as Tie-2 kinase inhibitors (Matloobi and Kappe, 2007; Chengguo et al., 2009), HIV-1 inhibitor (Gadhachanda et al., 2007; Naeem et al., 2009), antimalarial (Ngoy et al., 2011; Khan et al., 2009), adenosine $\mathrm{A}_{1}$ receptor antagonist (Chang et al., 2004), anticancer (Capdeville et al., 2002), analgesic (Rezvani and Shariati, 2010), cardiovascular (Atwal, 1988 and Hasanuzzaman et al., 2010) and antiallergic (Ozeki et al., 1989; Dahmardeh, 2011) activities. On the other hand, the importance of the pyridine ring in the chemistry of biological system has been greatly realized because of their presence as substructure in many natural products of therapeutic importance, involved in oxidation-reduction process. The potent biological activity of various vitamins and drugs (Joule and Mills, 2000; Henry, 2004; Li et al., 1999; Vacher et al., 1999; Nasratun et al., 2009) is primarily contributed by the presence of pyridine ring in their molecular make-up. Furthermore, the pyridine ring is found in the skeleton of many compounds with potent antibacterial, antifungal and anticancer properties (Millet et al., 2004; Mallea et al., 2003; Abou-Ghalia and Amr, 2004; Amr et al., 2009; Jill et al., 2011). In view of these reports and in continuation of our previous work in heterocyclic chemistry, we herein synthesized some new derivatives containing heterocyclic ring fused with $\mathrm{N}$ methylpiperdion and/or pyrido [4,3-d] pyrimidine

Corresponding Author: Abdel-Galil E. Amr, Department of Pharmaceutical Chemistry, College of Pharmacy,

King Saud University, Saudi Arabia 
Am. J. Biochem. \& Biotech., 7 (2): 43-54, 2011

structure for the evaluation of their anticancer activities. In view of a beforementioned biological activities and as a part of our interest in the sereach for novel anticancer agents, we report herein the synthesis of several thiazolo [3, 2-a] pyrido [4, 3-d] pyrimidine derivatives and evaluate of their anticancer activities.

\section{MATERIALS AND METHODS}

Chemistry: All melting points were determined on open glass capillaries using an Electrothermal IA 9000 digital melting point apparatus and are uncorrected. Elemental analyses were performed on Elementar, Vario El, Microanalytical Unit, National Research Centre, Cairo, Egypt and were found win $\pm 0.4 \%$ of the theoretical values. Infrared (IR) spectra were recorded on Carlzeise Spectrophotometer model 'UR 10' spectrophotometer using the $\mathrm{KBr}$ disc technique. ${ }^{1} \mathrm{H}$ NMR spectra were recorded on Varian Gemini 270 $\mathrm{MHz}$ spectrometer (DMSO- $\mathrm{d}_{6}$ or $\mathrm{CDCl}_{3}$ ) and the chemical shift are given in $\delta$ (parts per million) downfield from Tetramethylsilane (TMS) as an internal standard. The Mass Spectra (MS) were measured using a Finnegan SSQ 7000 mass spectrometer. The anticancer screening occurred in United States National Institute of Health (NIH)/National Cancer Institute (NCI). The starting material, 3,5bisarylmethylene-1-methyl-4-piperidon (1) was synthesized according to the reported procedures (Lyle et al., 1973; Mcelvain and Rorig, 1948; AbdelLatif and Lamiaa, 2010).

Synthesis of thiopyrimidene derivatives (2a-e): To a solution of 1a-e (0.01 mile) in $25 \mathrm{ml}$ absolute ethanol, $0.5 \mathrm{~g}$ potassium hydroxide and thiourea $(0.76 \mathrm{~g}, 0.01$ moles) were added. The reaction mixture was refluxed for $3 \mathrm{hrs}$. Left to cooland poured gradually onto cold water. The solid formed was filtered off, washed with water and crystallized from the proper solvent to give pyrimidin-2- $(1 \mathrm{H})$ thiones $2 \mathrm{a}-\mathrm{e}$, respectively.

8-Benzylidene-3,4,5,6,7,8-hexahydro-6-methyl-4phenylpyrido[4,3-d]pyrimidine-2(1H)-thione (2a): Yield 89\%, mp $190-193^{\circ} \mathrm{C}$; IR (KBr) cm${ }^{-1}$ : 3194, 3502; ${ }^{1} \mathrm{H}$ NMR (DMSO-d $): 2.20\left(\mathrm{~s}, 3 \mathrm{H}, \mathrm{CH}_{3}\right), 2.85(\mathrm{~m}, 2 \mathrm{H}$, H-5), 3.10-3.19 (dd, 2H, J = 7.0 Hz, J = 5.0 Hz, H-7), $4.95(\mathrm{~s}, 1 \mathrm{H}, \mathrm{H}-4), 7.15-7.40(\mathrm{~m}, 11 \mathrm{H}, \mathrm{Ar}-\mathrm{H}+\mathrm{C}=\mathrm{CH})$, 9.18, $9.48\left(2 \mathrm{~s}, 2 \mathrm{H}, 2 \mathrm{NH}\right.$, exchangeable with $\left.\mathrm{D}_{2} \mathrm{O}\right) ;{ }^{13} \mathrm{C}$ NMR: $44.48\left(\mathrm{CH}_{3}\right), 53.91(\mathrm{CH}$, pyrimidine $), 125.63$ (CH, methylene), 54.35, 54.39 (2 $\mathrm{CH}_{2}$-pyridine), 137.92 (C-pyridine), 138.26, 143.30, 180.6 (3C-pyrimidine), 126.65, 126.92, 128.13, 128.62, 128.74, 129.49, 136.19, 143.29 (12C, Ar-C); MS (EI): m/z $347\left[\mathrm{M}^{+}\right]$(80), 346 $\left(\mathrm{M}^{+}-\mathrm{H}^{+}\right)$(100), $214\left(346-\mathrm{C}_{6} \mathrm{H}_{5} \mathrm{CH}=\mathrm{NH}\right)(52), 254\left(\mathrm{M}^{+}-\right.$ $\mathrm{C}_{6} \mathrm{H}_{5}-\mathrm{H}^{+}$) (19). Anal. Calcd. for $\mathrm{C}_{21} \mathrm{H}_{21} \mathrm{~N}_{3} \mathrm{~S}$ : C, 72.58;
H, 6.1; N, 12.1; S, 9.22. Found: C, 72.56; H, 6.12; N, $12.07 ; \mathrm{S}, 9.25$.

8-(4-Flourobenzylidene)-3,4,5,6,7,8-hexahydro-6methyl-4-(4-flourophenyl)pyrido[4,3-d]pyrimidine2(1H)-thione (2b): Yield 91\%; mp $155-157^{\circ} \mathrm{C}$; IR $(\mathrm{KBr}) \mathrm{cm}^{-1}$ : 3204, $3344(\mathrm{NH}) ;{ }^{1} \mathrm{H}$ NMR (DMSO-d 6 ): $2.20\left(\mathrm{~s}, 3 \mathrm{H}, \mathrm{CH}_{3}\right), 2.41-2.60(\mathrm{~m}, 2 \mathrm{H}, \mathrm{H}-5), 3.10-3.20$ $(\mathrm{dd}, 2 \mathrm{H}, \mathrm{J}=7.0 \mathrm{~Hz}, \mathrm{~J}=5.0 \mathrm{~Hz}, \mathrm{H}-7), 4.96(\mathrm{~s}, 1 \mathrm{H}, \mathrm{H}-4)$, 7.11-7.50 (m, 9H, Ar-H+C = CH), 9.21, $9.51(2 \mathrm{~s}, 2 \mathrm{H}$, 2NH, exchangeable with $\left.\mathrm{D}_{2} \mathrm{O}\right) ;{ }^{13} \mathrm{C}$ NMR: $44.47\left(\mathrm{CH}_{3}\right)$, $53.91(\mathrm{CH}$, pyrimidine $), 50.93,52.71\left(2 \mathrm{CH}_{2}\right.$-pyridine $)$, 124.19 ( $\mathrm{CH}$, methylene), 136.81 (C-pyridine), 126.29, 126.47, 128.14, 128.61, 128.75, 128.89, 135.43, 143.33 (12C, Ar-C), 137.28, 140.41, 180.56 (3C-pyrimidine); MS (EI): m/z $383\left[\mathrm{M}^{+}\right]$(100), $339\left(\mathrm{M}^{+}-\mathrm{C}=\mathrm{S}\right)(11), 259$ (383-F- $\left.\mathrm{C}_{6} \mathrm{H}_{4} \mathrm{CH}=\mathrm{NH}\right) \quad(71)$; Anal. Calcd. For $\mathrm{C}_{21} \mathrm{H}_{19} \mathrm{~N}_{3} \mathrm{SF}_{2}$ : C, 65.77; H, 4.99; N, 10.96; S, 8.36. Found: C, 65.75; H, 5.01; N, 10.94; S, 8.38.

8-(2-Chlorobenzylidene)-3,4,5,6,7,8-hexahydro-6methyl-4-(2-chlorophenyl)pyrido[4,3-d]pyrimidine2(1H)-thione (2c): Yield 82\%; mp 200-202 ${ }^{\circ} \mathrm{C}$; IR $(\mathrm{KBr}) \mathrm{cm}^{-1}: 3351,3304(\mathrm{NH}) ;{ }^{1} \mathrm{H}$ NMR $\left(\mathrm{DMSO}^{-} \mathrm{d}_{6}\right)$ : $2.20\left(\mathrm{~s}, 3 \mathrm{H}, \mathrm{CH}_{3}\right), 2.50-2.71(\mathrm{~m}, 2 \mathrm{H}, \mathrm{H}-5), 3.21-3.30$ $(\mathrm{dd}, 2 \mathrm{H}, \mathrm{J}=7.0 \mathrm{~Hz}, \mathrm{~J}=5.0 \mathrm{~Hz}, \mathrm{H}-7), 5.41$ (s, 1H, H-4), 7.15-7.55 (m, 9H, Ar-H + C = CH), 9.10, $9.71(2 \mathrm{~s}, 2 \mathrm{H}$, 2NH, exchangeable with $\left.\mathrm{D}_{2} \mathrm{O}\right) ;{ }^{13} \mathrm{C}$ NMR: $44.44\left(\mathrm{CH}_{3}\right)$, 50.98, $52.73\left(2 \mathrm{CH}_{2}\right.$-pyridine $), 53.91(\mathrm{CH}$, pyrimidine $)$, 125.63 (CH, methylene), 126.31, 126.52, 128.19, 128.62, 128.74, 128.89, 135.43, 143.33 (12C, Ar-C), 134.22 (Cpyridine), 137.32, 140.42, 180.59 (3C-pyrimidine); MS (EI): m/z $416\left[\mathrm{M}^{+}\right](50), 417\left[\mathrm{M}^{+}+2\right](35), 419\left[\mathrm{M}^{+}+4\right]$ (8), $275\left(\mathrm{M}^{+}-\mathrm{Cl}-\mathrm{C}_{6} \mathrm{H}_{4} \mathrm{CH}=\mathrm{NH},-\mathrm{H}_{2}\right)$ (100); Anal. Calcd. for $\mathrm{C}_{21} \mathrm{H}_{19} \mathrm{~N}_{3} \mathrm{SCl}_{2}: \mathrm{C}, 60.57 ; \mathrm{H}, 4.6 ; \mathrm{N}, 10.1 ; \mathrm{S}, 7.7$. Found: C, 60.6; H, 4.57; N, 10.07; S, 7.71.

8-(4-Chlorobenzylidene)-3,4,5,6,7,8-hexahydro-6methyl-4-(4-chlorophenyl)-pyrido[4,3-d]pyrimidine2(1H)-thione (2d): Yield $88 \%$; mp 202-205 ${ }^{\circ} \mathrm{C}$; IR $(\mathrm{KBr}) \mathrm{cm}^{-1}: 3176,3248(\mathrm{NH}) ;{ }^{1} \mathrm{H}$ NMR (DMSO-d 6 ): $2.32\left(\mathrm{~s}, 3 \mathrm{H}, \mathrm{CH}_{3}\right), 2.42-2.63(\mathrm{~m}, 2 \mathrm{H}, \mathrm{H}-5), 3.31-3.43$ $(\mathrm{dd}, 2 \mathrm{H}, \mathrm{J}=7.0 \mathrm{~Hz}, \mathrm{~J}=5.0 \mathrm{~Hz}, \mathrm{H}-7), 5.02$ (s, 1H, H-4), 7.10-7.60 (m, 9H, Ar-H + C=CH), 9.10, $9.60(2 \mathrm{~s}, 2 \mathrm{H}$, $2 \mathrm{NH}$, exchangeable with $\left.\mathrm{D}_{2} \mathrm{O}\right) ;{ }^{13} \mathrm{C}$ NMR: $44.48\left(\mathrm{CH}_{3}\right)$, 50.96, 54.74 (2 $\mathrm{CH}_{2}$-pyridine), $53.91(\mathrm{CH}$, pyrimidine $)$, 124.28 (CH, methylene), 126.42, 126.50, 128.15, $128.61,128.79,128.89,135.43,143.37$ (12C, Ar-C), 137.36, 140.46, 180.82 (3C-pyrimidine), 136.88 (Cpyridine); MS (EI): m/z $416\left[\mathrm{M}^{+}\right](87), 417\left(\mathrm{M}^{+}+2\right)$ (59), $419\left(\mathrm{M}^{+}+4\right)(13), 414\left(\mathrm{M}^{+}-\mathrm{H}_{2}\right)(100), 275\left(\mathrm{M}^{+}-\mathrm{Cl}-\right.$ $\left.\mathrm{C}_{6} \mathrm{H}_{4} \mathrm{CH}=\mathrm{NH}, \quad-\mathrm{H}_{2}\right) \quad$ (72); Anal. Calcd. for 
$\mathrm{C}_{21} \mathrm{H}_{19} \mathrm{~N}_{3} \mathrm{SCl}_{2}$ : C, 60.57; H, 4.6; N, 10.1; S, 7.7. Found: C, $60.55 ; \mathrm{H}, 4.62 ; \mathrm{N}, 10.13 ; \mathrm{S}, 7.68$.

8-(3-Bromobenzylidene)-3,4,5,6,7,8-hexahydro-6methyl-4-(3-bromophenyl)pyrido[4,3-d]pyrimidine2(1H)-thione (2e): Yield 85\%; mp $180-182^{\circ} \mathrm{C}$; IR $(\mathrm{KBr}) \mathrm{cm}^{-1}$ : 3346, $3275(\mathrm{NH}) ;{ }^{1} \mathrm{H}$ NMR (DMSO-d $)_{6}$ ): $2.23\left(\mathrm{~s}, 3 \mathrm{H}, \mathrm{CH}_{3}\right), 2.32-2.53(\mathrm{~m}, 2 \mathrm{H}, \mathrm{H}-5), 3.32-3.41$ $(\mathrm{dd}, 2 \mathrm{H}, \mathrm{J}=7.0 \mathrm{~Hz}, \mathrm{~J}=5.0 \mathrm{~Hz}, \mathrm{H}-7), 5.31(\mathrm{~s}, 1 \mathrm{H}, \mathrm{H}-$ 4), 7.30-7.60 (m, 9H, Ar-H+C=CH), 9.01, $9.42(2 \mathrm{~s}, 2 \mathrm{H}$, 2NH, exchangeable with $\left.\mathrm{D}_{2} \mathrm{O}\right) ;{ }^{13} \mathrm{C}$ NMR: $44.49\left(\mathrm{CH}_{3}\right)$, 50.95, $52.74\left(2 \mathrm{CH}_{2}\right.$-pyridine $), 53.74(\mathrm{CH}$, pyrimidine), 124.23 (CH, methylene), 126.31, 126.48, 128.21, $128.71,128.68,128.87,135.45,143.37$ (12Ar-C), 136.84 (C-pyridine), 137.29, 140.44, 180.57 (3Cpyrimidine). MS (EI): m/z $505\left[\mathrm{M}^{+}\right](47), 507\left(\mathrm{M}^{+}+2\right)$ (95), $509\left(\mathrm{M}^{+}+4\right)$ (45), $349\left(\mathrm{M}^{+}-\mathrm{Br}-\mathrm{C}_{6} \mathrm{H}_{4}\right)$ (100); Anal. Calcd. For $\mathrm{C}_{21} \mathrm{H}_{19} \mathrm{~N}_{3} \mathrm{SBr}_{2}$ : C, 49.91; H, 3.79; N, 3.18; S, 6.34. Found: C, 49.91; H, 3.79; N, 3.18; S, 6.34.

Synthesis of 5-aryl-9-arylmethylene-2,3,6,7,8,9hexahydro-7-methy-5H-thiazolo[3,2-a]pyrido-[4,3-

d]pyrimidines (3a-e): To a solution of 1a-e (0.02 mole), in a mixture of butanol $(100 \mathrm{~mL})$, DMSO (40 ml)and 2-aminothiazoline $(2.7 \mathrm{~g}, 0.023$ mole $)$ was added. The reaction mixture was refluxed for $24 \mathrm{hrs}$, the solvent was concentrated under reduced pressureand the residue was solidified with water, the formed solid was filtered offand crystallized from the proper solvent to give thiazolo[3,2-a]pyrido[4,3d]pyrimidines $3 \mathrm{a}-\mathrm{e}$, respectively.

9-Benzylidene-2,3,6,7,8,9-hexahydro-7-methyl-5H-5phenyl-thiazolo[3,2-a]pyrido[4,3-d]-pyrimidine (3a): Yield $81 \%$; mp $169-171^{\circ} \mathrm{C}$; IR (KBr) cm $\mathrm{cm}^{-1}: 1635$ $(\mathrm{C}=\mathrm{N}) ;{ }^{1} \mathrm{H}$ NMR $\left(\mathrm{DMSO}-\mathrm{d}_{6}\right): 2.10\left(\mathrm{~s}, 2 \mathrm{H}-\mathrm{C}_{6}\right), 2.71$ (s, $\left.3 \mathrm{H}, \mathrm{CH}_{3}\right), 2.99-3.01$ (m, 2H-C-2), 3.05-3.15 (m, 2H$\left.\mathrm{C}_{3}\right), 3.60-3.58\left(\mathrm{~m}, 2 \mathrm{H}-\mathrm{C}_{8}\right), 5.51\left(\mathrm{~s}, 1 \mathrm{H}-\mathrm{C}_{5}\right), 7.36-6.57$ $(\mathrm{m}, 11 \mathrm{H}, \mathrm{Ar}-\mathrm{H}+\mathrm{CH}=\mathrm{C}) ;{ }^{13} \mathrm{C}$ NMR: 24.61, 50.35 $\left(2 \mathrm{CH}_{2}\right.$, thiazole $), 44.31\left(\mathrm{CH}_{3}\right), 51.71,53.41\left(2 \mathrm{CH}_{2}-\right.$ pyridine), $58.31(\mathrm{CH}$, pyrimidine $), 124.28(\mathrm{CH}$, methylene), 126.42, 127.22, 128.12, 128.65, 128.72, 135.32, 138.32 (12C, Ar-C), 129.21, 134.82, 183.66 (3Cpyrimidine), 137.1 (C-pyridine). MS (EI) m/z (\%): 373 $\left[\mathrm{M}^{+}\right]$(79), $262\left(\mathrm{M}^{+}-\mathrm{ClC}_{6} \mathrm{H}_{4}\right)(100), 249$ (262-CH) (50); Anal. Calcd. for $\mathrm{C}_{23} \mathrm{H}_{23} \mathrm{~N}_{3} \mathrm{~S}$ : C,73.95; H, 6.20; N, 11.25; S, 8.58. Found: C,73.93; H, 6.22; N, 11.27; S, 8.56.

9-(4-Fluorobenzylidene)-2,3,6,7,8,9-hexahydro-7methyl-5H-5-(4-flourophenyl)-thiazolo[3,2a]pyrido[4,3-d]pyrimidine (3b): Yield $82 \%$; mp 132$134^{\circ} \mathrm{C}$; IR $(\mathrm{KBr}) \mathrm{cm}^{-1}: 1631(\mathrm{C}=\mathrm{N})$; ${ }^{1} \mathrm{H}$ NMR (DMSO$\left.\mathrm{d}_{6}\right): 2.10\left(\mathrm{~s}, 2 \mathrm{H}-\mathrm{C}_{6}\right), 2.72\left(\mathrm{~s}, 3 \mathrm{H}, \mathrm{CH}_{3}\right), 2.99-3.11(\mathrm{~m}$,
2H-C-2), 3.21-3.25 (m 2H-C $), 3.61-3.68$ (m, 2H-C $)$, $5.52\left(\mathrm{~s}, 1 \mathrm{H}-\mathrm{C}_{5}\right), 7.28-6.56(\mathrm{~m}, 9 \mathrm{H}, \mathrm{Ar}-\mathrm{H}+\mathrm{CH}=\mathrm{C}) ;{ }^{13} \mathrm{C}$ NMR: $24.59,50.33\left(2 \mathrm{CH}_{2}\right.$, thiazole $), 44.29\left(\mathrm{CH}_{3}\right)$, 51.69, $53.40\left(2 \mathrm{CH}_{2}\right.$-pyridine $), 58.30(\mathrm{CH}$, pyrimidine $)$, $115.11,115.32,128.11,129.41,130.41,134.30,162.23$ (12C, Ar-C), 124.28 (CH, methylene), 136.9 (Cpyridine), 129.19, 134.80, 183.64 (3C-pyrimidine); MS (EI): m/z $409\left[\mathrm{M}^{+}\right]$(100), $408\left(\mathrm{M}^{+}-\mathrm{H}\right)(97), 314\left(\mathrm{M}^{+}-\mathrm{F}-\right.$ $\mathrm{C}_{6} \mathrm{H}_{4}$ ) (70); Anal. Calcd. for $\mathrm{C}_{23} \mathrm{H}_{21} \mathrm{~N}_{3} \mathrm{SF}_{2}$ : C, 67.46; $\mathrm{H}$, 5.16; N, 10.26; S, 7.83. Found: C, 67.48; H, 5.14; N, $10.28 ; \mathrm{S}, 7.80$.

9-(2-Chlorobenzylidene)-2,3,6,7,8,9-hexahydro-7methyl-5H-5-(2-chlorophenyl)-thiazolo[3,2a]pyrido[4,3-d]pyrimidine (3c): Yield 79\%; mp 143$145^{\circ} \mathrm{C}$; IR $(\mathrm{KBr}) \mathrm{cm}^{-1}: 1636(\mathrm{C}=\mathrm{N}) ;{ }^{1} \mathrm{H}$ NMR (DMSO$\left.\mathrm{d}_{6}\right): 2.14\left(\mathrm{~s}, 2 \mathrm{H}-\mathrm{C}_{6}\right), 2.73\left(\mathrm{~s}, 3 \mathrm{H}, \mathrm{CH}_{3}\right), 2.98-3.09(\mathrm{~m}$, $2 \mathrm{H}-\mathrm{C}-2), 3.18-3.21\left(\mathrm{~m}, 2 \mathrm{H}-\mathrm{C}_{3}\right), 3.59-3.66\left(\mathrm{~m}, 2 \mathrm{H}-\mathrm{C}_{8}\right)$, $5.54\left(\mathrm{~s}, 1 \mathrm{H}-\mathrm{C}_{5}\right), 6.85-7.32(\mathrm{~m}, 9 \mathrm{H}, \mathrm{Ar}-\mathrm{H}+\mathrm{CH}=\mathrm{C}) ;{ }^{13} \mathrm{C}$ NMR: $24.59,50.33\left(2 \mathrm{CH}_{2}\right.$, thiazole $), 44.29\left(\mathrm{CH}_{3}\right)$, 51.69, 53.40 (2 $\mathrm{CH}_{2}$-pyridine $), \quad 58.33 \quad(\mathrm{CH}$, pyrimidine), 124.28 ( $\mathrm{CH}$, methylene), 115.21, 115.32, $128.30,129.81,130.52,134.32,162.20$ (12C, Ar-C), 136.91 (C-pyridine), 129.21, 134.80, 183.64 (3Cpyrimidine); MS (EI): m/z $442\left[\mathrm{M}^{+}\right](64), 444\left(\mathrm{M}^{+}+2\right)$ (40), $446\left(\mathrm{M}^{+}+4\right)(6), 406\left(\mathrm{M}^{+}-\mathrm{Cl}\right)(17), 362\left(\mathrm{M}^{+}-\right.$ $\mathrm{CH}_{2}=\mathrm{NCH}_{3},-\mathrm{H}^{+}$) (18), 44 (100); Anal. Calcd. for $\mathrm{C}_{23} \mathrm{H}_{21} \mathrm{~N}_{3} \mathrm{SCl}_{2}$ : C, 62.43; H, 4.78; N, 9.50; S, 7.24. Found: C, 62.41; H, 4.80; N, 9.58; S, 7.21.

9-(4-Chlorobenzylidene)-2,3,6,7,8,9-hexahydro-7methyl-5H-5-(4-chlorophenyl)-thiazolo-[3,2a]pyrido[4,3-d]pyrimidine (3d): Yield $80 \%$; mp 187$189^{\circ} \mathrm{C}$; IR $(\mathrm{KBr}) \mathrm{cm}^{-1}: 1638(\mathrm{C}=\mathrm{N})$; ${ }^{1} \mathrm{H}$ NMR (DMSO$\left.\mathrm{d}_{6}\right): 2.13\left(\mathrm{~s}, 2 \mathrm{H}-\mathrm{C}_{6}\right), 2.72\left(\mathrm{~s}, 3 \mathrm{H}, \mathrm{CH}_{3}\right), 2.97-3.08(\mathrm{~m}$, $2 \mathrm{H}-\mathrm{C}_{-2}$ ), 3.16-3.19 (m 2H-C ${ }_{3}$ ), 3.58-3.65 (m, 2H-C ${ }_{8}$ ), $5.53\left(\mathrm{~s}, 1 \mathrm{H}-\mathrm{C}_{5}\right), 6.84-7.31(\mathrm{~m}, 9 \mathrm{H}, \mathrm{Ar}-\mathrm{H}+\mathrm{CH}=\mathrm{C}) ;{ }^{13} \mathrm{C}$ NMR: $24.63,50.31\left(2 \mathrm{CH}_{2}\right.$, thiazole $), 44.24\left(\mathrm{CH}_{3}\right)$, 51.72, $53.41\left(2 \mathrm{CH}_{2}\right.$-pyridine $), 58.32(\mathrm{CH}$, pyrimidine $)$, $115.12,115.35,128.13,129.73,130.43,134.32,162.25$ (12C, Ar-C), 124.27 (CH, methylene), 136.85 (Cpyridine), 129.22, 134.81, 183.50 (3C-pyrimidine); MS (EI): $\mathrm{m} / \mathrm{z} 442\left[\mathrm{M}^{+}\right](44), 444\left(\mathrm{M}^{+}+2\right)(16), 446\left(\mathrm{M}^{+}+4\right)$ (6), $318\left(\mathrm{M}^{+}-\mathrm{CH}-\mathrm{C}_{6} \mathrm{H}_{4}-\mathrm{Cl}\right)$ (57), 113 (100); Anal. Calcd. for $\mathrm{C}_{23} \mathrm{H}_{21} \mathrm{~N}_{3} \mathrm{SCl}_{2}$ : C, 62.43; H, 4.78; N, 9.50; S, 7.24. Found: C, 62.45; H, 4.76; N, 9.52; S, 7.22.

9-(3-Bromobenzylidene)-2,3,6,7,8,9-hexahydro-7methyl-5H-5-(3-bromophenyl)-thiazolo-[3,2a]pyrido[4,3-d]pyrimidine (3e): Yield 78\%; mp 139$142^{\circ} \mathrm{C}$; IR $(\mathrm{KBr}) \mathrm{cm}^{-1}$ : $1634(\mathrm{C}=\mathrm{N})$; ${ }^{1} \mathrm{H}$ NMR (DMSO$\left.\mathrm{d}_{6}\right): 2.15\left(\mathrm{~s}, 2 \mathrm{H}-\mathrm{C}_{6}\right), 2.73\left(\mathrm{~s}, 3 \mathrm{H}, \mathrm{CH}_{3}\right), 2.99-3.10(\mathrm{~m}$, $2 \mathrm{H}-\mathrm{C}-2), 3.19-3.21$ (m 2H-C 3 ), 3.65-3.71 (m, $\left.2 \mathrm{H}-\mathrm{C}_{8}\right)$, 
$5.56\left(\mathrm{~s}, 1 \mathrm{H}-\mathrm{C}_{5}\right), 6.66-7.28(\mathrm{~m}, 9 \mathrm{H}, \mathrm{Ar}-\mathrm{H}+\mathrm{CH}=\mathrm{C}) ;{ }^{13} \mathrm{C}$ NMR: $24.64,50.32\left(2 \mathrm{CH}_{2}\right.$, thiazole $), 44.25\left(\mathrm{CH}_{3}\right)$, 51.70, $53.40\left(2 \mathrm{CH}_{2}\right.$-pyridine $), 58.33(\mathrm{CH}$, pyrimidine $)$, 115.14, 115.34, 128.11, 129.76, 130.45, 134.34, 162.27 (12C, Ar-C), 124.25 (CH, methylene), 136.86 (Cpyridine), 129.20, 134.80, 183.55 (3C-pyrimidine); MS (EI): m/z $531\left[\mathrm{M}^{+}\right](45), 533\left(\mathrm{M}^{+}+2\right)(91), 535\left(\mathrm{M}^{+}+4\right)$ (44), 451 ( $\left.\mathrm{M}^{+}-\mathrm{Br}\right)$ (100); Anal. Calcd. for $\mathrm{C}_{23} \mathrm{H}_{21} \mathrm{~N}_{3} \mathrm{SBr}_{2}$ : C, 51.99; H, 3.98; N, 7.91; S, 6.03. Found: C, 51.97; H, 4.01; N, 7.89; S, 6.05.

Synthesis of 5-aryl-9-arylmethylene-6,7,8,9tetrahydro-7-methyl-2H-thiazolo[3,2-a]pyrido-[4,3d]pyrimidin-3(5H)-ones (4a-e): To a mixture of $2 \mathrm{a}-\mathrm{e}$ (0.01 mole), chloroacetic acid ( $1 \mathrm{~g}, 0.01$ mole), $6 \mathrm{~g}$ of fused sodium acetate in $30 \mathrm{ml}$ of glacial acetic acid and $15 \mathrm{~mL}$ of acetic anhydride was refluxed for $3 \mathrm{hrs}$., poured gradually onto cold water. The solid formed was filtered offand crystallized from the proper solvent to give pyrido[4,3-d]pyrimidinones 4a-e, respectively.

\section{9-Benzylidene-6,7,8,9-tetrahydro-7-methyl-5-} phenyl-2H-thiazolo[3,2-a]pyrido[4,3-d]pyrimidine3(5H)-one (4a): Yield 69\%; m.p 214-216 ${ }^{\circ} \mathrm{C}$; IR (KBr) $\mathrm{cm}^{-1}: 1734(\mathrm{C}=\mathrm{O}) ;{ }^{1} \mathrm{H}$ NMR (DMSO-d $\left.{ }_{6}\right): 2.21(\mathrm{~s}, 3 \mathrm{H}$, $\mathrm{CH}_{3}$ ), 2.62 (s, 2H-C $), 3.70-4.02$ (m, 2H-C $), 4.12$ (s, 2H$\left.\mathrm{C}_{-}\right), 5.81\left(\mathrm{~s}, 1 \mathrm{H}-\mathrm{C}_{5}\right), 7.33-7.52(\mathrm{~m}, 10 \mathrm{H}, \mathrm{Ar}-\mathrm{H}), 7.72(\mathrm{~s}$, 1H, $\mathrm{CH}=\mathrm{C}) ;{ }^{13} \mathrm{C}$ NMR: $30.61\left(\mathrm{CH}_{2}\right.$, thiazole $), 44.21\left(\mathrm{CH}_{3}\right)$, $45.31\left(\mathrm{CH}\right.$, pyrimidine), 51.41, $53.43\left(2 \mathrm{CH}_{2}\right.$-pyridine $)$, 126.42, 126.82, 127.32, 128.11, 128.63, 128.73, 135.32, 143.22 (12C, Ar-C), 124.23 (CH, methylene), 136.91 (Cpyridine), 129.65, 134.84, 163.66 (3C-pyrimidine), 171.11 $(\mathrm{C}=\mathrm{O})$; MS (EI): m/z $387\left[\mathrm{M}^{+}\right](44), 386\left(\mathrm{M}^{+}-\mathrm{H}^{+}\right)(100)$, 312 (386- $\left.\mathrm{SCH}_{2}-\mathrm{C}=\mathrm{O}\right)$ (7); Anal. Calcd. for $\mathrm{C}_{23} \mathrm{H}_{21} \mathrm{~N}_{3} \mathrm{SO}$ : C, 71.28; H, 5.46; N, 10.84; S, 8.27. Found: C, 71.30; H, $5.43 ; \mathrm{N}, 10.82 ; \mathrm{S}, 8.29$.

\section{9-(4-Flourobenzylidene)-6,7,8,9-tetrahydro-7-} methyl-5-(4-flourophenyl)-2H-thiazolo[3,2a]pyrido[4,3-d]pyrimidine-3(5H)-one (4b): Yield 76\%; mp 203-205 ${ }^{\circ}$; IR ( $\left.\mathrm{KBr}\right) \mathrm{cm}^{-1}: 1732(\mathrm{C}=\mathrm{O}) ;{ }^{1} \mathrm{H}$ NMR (DMSO-d $\left.)_{6}\right): 2.22\left(\mathrm{~s}, 3 \mathrm{H}, \mathrm{CH}_{3}\right), 2.60(\mathrm{~s}, 2 \mathrm{H}-$ $\left.\mathrm{C}_{6}\right), 3.79-4.11\left(\mathrm{~m}, 2 \mathrm{H}-\mathrm{C}_{8}\right), 4.31$ (s, 2H-C-2), 6.31 (s, 1H$\left.\mathrm{C}_{5}\right)$, 7.31-7.50 (m, 8H, Ar-H), $7.70(\mathrm{~s}, 1 \mathrm{H}, \mathrm{CH}=\mathrm{C}) ;{ }^{13} \mathrm{C}$ NMR: $30.62\left(\mathrm{CH}_{2}\right.$, thiazole $), 44.23\left(\mathrm{CH}_{3}\right), 45.33(\mathrm{CH}$, pyrimidine), 51.40, $53.41\left(2 \mathrm{CH}_{2}\right.$-pyridine $), 126.43$, $126.83,127.30,128.31,128.61,128.75,135.22,143.10$ (12C, Ar-C), 124.25 (CH, methylene), 129.28, 134.81, 163.70 (3C-pyrimidine), 136.91 (C-pyridine), 170.89 $(\mathrm{C}=\mathrm{O}) ; \mathrm{MS}(\mathrm{EI}): \mathrm{m} / \mathrm{z} 423\left[\mathrm{M}^{+}\right](100), 422\left(\mathrm{M}^{+}-\mathrm{H}^{+}\right)$ (92), $328\left(\mathrm{M}^{+}-\mathrm{F}_{-} \mathrm{C}_{6} \mathrm{H}_{4}\right)$ (71); Anal. Calcd. for $\mathrm{C}_{23} \mathrm{H}_{19} \mathrm{~N}_{3} \mathrm{SOF}_{2}$ : C, 65.23; H, 4.52; N, 9.92; S, 7.57. Found: C, 65.21; H, 4.54; N, 9.90; S, 7.59.
9-(2-Chlorobenzylidene)-6,7,8,9-tetrahydro-7methyl-5-(2-chlorophenyl)-2H-thiazolo[3,2a]pyrido[4,3-d]pyrimidine-3(5H)-one (4c): Yield 71\%; mp 210-212 ${ }^{\circ} \mathrm{C}$; IR $(\mathrm{KBr}) \mathrm{cm}^{-1}$ : $1736(\mathrm{C}=\mathrm{O}) ;{ }^{1} \mathrm{H}$ NMR (DMSO-d $\left.\mathrm{d}_{6}\right): 2.26\left(\mathrm{~s}, 3 \mathrm{H}, \mathrm{CH}_{3}\right), 2.62\left(\mathrm{~s}, 2 \mathrm{H}-\mathrm{C}_{6}\right)$, 3.81-4.17 (m, 2H-C $\left.{ }_{8}\right), 4.21$ (s, 2H-C-2), 6.33 (s, 1H-C ${ }_{5}$ ), 7.33-7.52 (m, 8H, Ar-H), $7.72(\mathrm{~s}, 1 \mathrm{H}, \mathrm{CH}=\mathrm{C}) ;{ }^{13} \mathrm{C}$ NMR: $30.64\left(\mathrm{CH}_{2}\right.$, thiazole), $44.25\left(\mathrm{CH}_{3}\right), 45.31(\mathrm{CH}$, pyrimidine), 51.42, $53.43\left(2 \mathrm{CH}_{2}\right.$-pyridine $), 124.25(\mathrm{CH}$, methylene), 126.41, 126.80, 127.31, 128.21, 128.59, $128.71,135.26,143.32$ (12C, Ar-C), 136.81 (Cpyridine), 129.26, 134.86, 163.55 (3C-pyrimidine), $171.22(\mathrm{C}=\mathrm{O}) ; \mathrm{MS}(\mathrm{EI}): \mathrm{m} / \mathrm{z} 456\left[\mathrm{M}^{+}\right]$(89), 444 $\left(\mathrm{M}^{+}+2\right)(62), 458\left(\mathrm{M}^{+}+4\right)(27), 420\left(\mathrm{M}^{+}-\mathrm{Cl}\right)(100)$; Anal. Calcd. for $\mathrm{C}_{23} \mathrm{H}_{19} \mathrm{~N}_{3} \mathrm{SOCl}_{2}$ : C, 60.50; H,4.19; N, 9.21; S, 7.02. Found: C, 60.54; H,4.17; N, 9.23; S, 7.00.

9-(4-Chlorobenzylidene)-6,7,8,9-tetrahydro-7methyl-5-(4-chlorophenyl)-2H-thiazolo[3,2a]pyrido[4,3-d]pyrimidine-3(5H)-one $\quad(\mathbf{4 d})$ : Yield 79\%; mp 232-234 ${ }^{\circ} \mathrm{C}$; IR (KBr) cm ${ }^{-1}: 1737(\mathrm{C}=\mathrm{O}) ;{ }^{1} \mathrm{H}$ NMR (DMSO-d $\left.{ }_{6}\right): 2.28\left(\mathrm{~s}, 3 \mathrm{H}, \mathrm{CH}_{3}\right), 2.61$ (s, 2H-C $)$, 3.83-4.09 (m, 2H-C $), 4.18$ (s, 2H-C-2), 5.90 (s, 1H-C $)$, 7.31-7.54 (m, 8H, Ar-H), $7.72(\mathrm{~s}, 1 \mathrm{H}, \mathrm{CH}=\mathrm{C}) ;{ }^{13} \mathrm{C}$ NMR: $30.62\left(\mathrm{CH}_{2}\right.$, thiazole), $44.26\left(\mathrm{CH}_{3}\right), 45.33(\mathrm{CH}$, pyrimidine), 51.41, $53.41\left(2 \mathrm{CH}_{2}\right.$-pyridine $), 124.21(\mathrm{CH}$, methylene), 126.43, 126.81, 127.42, 128.18, 128.53, 128.73, 135.28, 143.30 (12C, Ar-C), 129.31, 134.85, 163.33 (3C-pyrimidine), 136.83 (C-pyridine), 171.00 $(\mathrm{C}=\mathrm{O}) ; \mathrm{MS}(\mathrm{EI}): \mathrm{m} / \mathrm{z} 456\left[\mathrm{M}^{+}\right](62), 444\left(\mathrm{M}^{+}+2\right)(62)$, $458\left(\mathrm{M}^{+}+4\right)(27), 420\left(\mathrm{M}^{+}-\mathrm{Cl}\right)(39), 458\left(\mathrm{M}^{+}+4\right)(7)$, $345 \quad\left(\mathrm{M}^{+}-\mathrm{Cl}-\mathrm{C}_{6} \mathrm{H}_{4}\right) \quad$ (100); Anal. Calcd. for $\mathrm{C}_{23} \mathrm{H}_{19} \mathrm{~N}_{3} \mathrm{SOCl}_{2}$ : C, 60.52; H, 4.19; N, 9.21; S, 7.02. Found: C, 60.50; H, 4.21; N, 9.19; S, 7.04.

\section{9-(4-Bromobenzylidene)-6,7,8,9-tetrahydro-7-} methyl-5-(4-bromophenyl)-2H-thiazolo[3,2a]pyrido[4,3-d]pyrimidine-3(5H)-one (4e): Yield 61\%; mp 167-169 ${ }^{\circ}$; IR $(\mathrm{KBr}) \mathrm{cm}^{-1}: 1735(\mathrm{C}=\mathrm{O}) ;{ }^{1} \mathrm{H}$ NMR (DMSO-d $\left.{ }_{6}\right): 2.27$ (s, 3H, $\left.\mathrm{CH}_{3}\right), 2.62\left(\mathrm{~s}, 2 \mathrm{H}-\mathrm{C}_{6}\right)$, 3.85-4.10 (m, 2H-C $), 4.19$ (s, 2H-C-2), 6.06 (s, 1H-C $)$, 7.29-7.58 (m, 8H, Ar-H), $7.68(\mathrm{~s}, 1 \mathrm{H}, \mathrm{CH}=\mathrm{C}) ;{ }^{13} \mathrm{C}$ NMR: $30.60\left(\mathrm{CH}_{2}\right.$, thiazole), $44.28\left(\mathrm{CH}_{3}\right), 45.30(\mathrm{CH}$, pyrimidine), 51.39, $53.43\left(2 \mathrm{CH}_{2}\right.$-pyridine $), 124.25(\mathrm{CH}$, methylene), 126.41, 126.82, 127.43, 128.16, 128.51, 128.72, 135.26, 143.31 (12C, Ar-C), 136.80 (Cpyridine), 129.33, 134.82, 163.35 (3C-pyrimidine), $171.32(\mathrm{C}=\mathrm{O}) ; \mathrm{MS}(\mathrm{EI}): \mathrm{m} / \mathrm{z} 545\left[\mathrm{M}^{+}\right](31), 547$ $\left(\mathrm{M}^{+}+2\right)(66), 549\left(\mathrm{M}^{+}+4\right)(28), 376\left(\mathrm{M}^{+}-\mathrm{BrC}_{6} \mathrm{H}_{4}=\mathrm{CH}^{+}\right)$ (100); Anal. Calcd. for $\mathrm{C}_{23} \mathrm{H}_{19} \mathrm{~N}_{3} \mathrm{SOBr}_{2}$ : C, 50.66; $\mathrm{H}$, 3.51; N, 7.70; S, 5.88. Found: C, 50.68; H, 3.53; N, $7.68 ; \mathrm{S}, 5.86$. 
Synthesis of 5-aryl-9-arylmethylene-6,7,8,9-tetrahydro-2,7-dimethyl-2H-thiazolo[3,2-a]-pyrido[4,3d]pyrimidin-3(5H)-ones (5a-e): A mixture of $2 \mathrm{a}-\mathrm{e}$ (0.01 mole), 2-bromopropionic acid (1.54g, 0.01 mole), $6 \mathrm{~g}$ of fused sodium acetate in $30 \mathrm{ml}$ of glacial acetic acid and $15 \mathrm{ml}$ of acetic anhydride was refluxed for $2 \mathrm{hrs}$, poured onto cold water. The solid formed was filtered offand crystallized from the proper solvent to give pyrido[4,3-d]pyrimidin-3 $(5 \mathrm{H})$-ones $5 \mathrm{a}$ e, respectively.

9-Benzylidene-6,7,8,9-tetrahydro-2,7-dimethyl-5phenyl-2H-thiazolo[3,2-a]pyrido[4,3-d]-pyrimidine3(5H)-one (5a): Yield 69\%; mp 216-218 ${ }^{\circ} \mathrm{C}$; IR (KBr) $\mathrm{cm}^{-1}: 1726(\mathrm{C}=\mathrm{O}) ;{ }^{1} \mathrm{H}$ NMR (DMSO-d 6 ): 2.12 (s, 2H$\mathrm{C}_{6}$ ), 2.71 (s, $3 \mathrm{H}, \mathrm{CH}_{3}$, pyridine ring), 3.65-4.22 (2d, $2 \mathrm{H}-$ $\mathrm{C}_{8}$ ), 4.51 (q, $\left.1 \mathrm{H}-\mathrm{C}_{-2}\right), 6.05$ (s, $\left.1 \mathrm{H}, \mathrm{CH}-\mathrm{C}_{5}\right), 1.41(\mathrm{~d}, 3 \mathrm{H}$, $\mathrm{CH}_{3}$, thiazole ring), 7.30-7.50 (m, $\left.11 \mathrm{H}, \mathrm{Ar}-\mathrm{H}+\mathrm{CH}=\mathrm{C}\right)$; ${ }^{13} \mathrm{C}$ NMR: $20.1\left(\mathrm{CH}_{3}\right.$, thiazole ring), $43.71(\mathrm{CH}$, thiazole), $44.21\left(\mathrm{CH}_{3}\right.$, pyridine ring), $45.61(\mathrm{CH}$, pyrimidine), 51.30, 53.40 ( $2 \mathrm{CH}_{2}$-pyridine), $124.28(\mathrm{CH}$, methylene), 126.41, 126.80, 127.00, 128.01, 128.64, $128.70,135.22,143.32$ (12C, Ar-C), 136.93 (Cpyridine), 129.12, 134.81, 163.16 (3C-pyrimidine), $176.21(C=\mathrm{O})$; MS (EI): m/z $401\left[\mathrm{M}^{+}\right](50), 400\left(\mathrm{M}^{+}-\right.$ $\left.\mathrm{H}^{+}\right)$(100), $312\left(\mathrm{M}^{+}-\mathrm{C}_{6} \mathrm{H}_{5}-\mathrm{CH}+\mathrm{H}^{+}\right)(9)$; Anal. Calcd. for $\mathrm{C}_{24} \mathrm{H}_{23} \mathrm{~N}_{3} \mathrm{SO}$ : C, 71.79; H, 5.77; N, 10.46; S, 7.98. Found: C, 71.81; H, 5.75; N, 10.46; S, 7.98.

9-(4-Flourobenzylidene)-2,7-dimethyl-6,7,8,9tetrahydro-5-(4-flourophenyl)-2H-thiazolo-[3,2a]pyrido[4,3-d]pyrimidine-3(5H)-one $\quad(5 \mathrm{~b})$ : Yield 81\%; mp 206-208 ${ }^{\circ} \mathrm{C}$; IR ( $\left.\mathrm{KBr}\right) \mathrm{cm}^{-1}: 1727(\mathrm{C}=\mathrm{O}) ;{ }^{1} \mathrm{H}$ NMR (DMSO-d $\left.\mathrm{d}_{6}\right): 1.43\left(\mathrm{~d}, 3 \mathrm{H}, \mathrm{CH}_{3}\right.$, thiazole ring), 2.10 (s, $\left.2 \mathrm{H}-\mathrm{C}_{6}\right), 2.72$ (s, 3H, $\mathrm{CH}_{3}$, pyridine ring), 3.63$4.20\left(2 \mathrm{~d}, 2 \mathrm{H}-\mathrm{C}_{8}\right), 4.53$ (q, 1H-C-2), 5.81 (s, 1H-C $)_{5}, 7.32-$ $7.60(\mathrm{~m}, 9 \mathrm{H}, \mathrm{Ar}-\mathrm{H}+\mathrm{CH}=\mathrm{C}) ;{ }^{13} \mathrm{C}$ NMR: $20.11\left(\mathrm{CH}_{3}\right.$, thiazole ring), $43.72(\mathrm{CH}$, thiazole $), 44.20\left(\mathrm{CH}_{3}\right.$, pyridine ring), 45.60 ( $\mathrm{CH}$, pyrimidine), 51.30, 53.41 (2 $\mathrm{CH}_{2}$-pyridine), $124.26(\mathrm{CH}$, methylene $), 126.40$, 126.80, 127.05, 128.01, 128.62, 128.72, 135.18, 143.36 (12C, Ar-C), 129.21, 134.61, 163.21 (3C-pyrimidine), 136.72 (C-pyridine), $176.0(C=\mathrm{O}) ; \mathrm{MS}(\mathrm{EI}): \mathrm{m} / \mathrm{z} 437$ $\left[\mathrm{M}^{+}\right](17), 436\left(\mathrm{M}^{+}-\mathrm{H}^{+}\right)(28), 341\left(436-\mathrm{F}-\mathrm{C}_{6} \mathrm{H}_{4}\right)(100)$; Anal. Calcd. for $\mathrm{C}_{24} \mathrm{H}_{21} \mathrm{~N}_{3} \mathrm{SOF}_{2}$ : C, 65.88; H, 4.83; N, 9.60; S, 7.32. Found: C, 65.90; H, 4.81; N, 9.58; S, 7.35.

9-(2-Chlorobenzylidene)-6,7,8,9-tetrahydro-2,7dimethyl-5-(2-chlorophenyl)-2H-thiazolo-[3,2a]pyrido[4,3-d]pyrimidine-3(5H)-one $\quad(5 \mathrm{c})$ : Yield 64\%; mp 219-221 ${ }^{\circ} \mathrm{C}$; IR (KBr) cm ${ }^{-1}: 1723(\mathrm{C}=\mathrm{O}) ;{ }^{1} \mathrm{H}$
NMR (DMSO- $\left.\mathrm{d}_{6}\right): 1.42\left(\mathrm{~d}, 3 \mathrm{H}, \mathrm{CH}_{3}\right.$, thiazole ring), 2.13 (s, $2 \mathrm{H}-\mathrm{C}_{6}$ ), 2.70 (s, 3H, $\mathrm{CH}_{3}$, pyridine ring), 3.65$4.22\left(2 \mathrm{~d}, 2 \mathrm{H}-\mathrm{C}_{8}\right), 4.51$ (q, 1H-C-2), 6.01 (s, 1H-C ${ }_{5}$ ), 7.22-7.65 (m, 9H, Ar-H+ $\mathrm{CH}=\mathrm{C}) ;{ }^{13} \mathrm{C}$ NMR: 20.21 $\left(\mathrm{CH}_{3}\right.$, thiazole ring), $43.71(\mathrm{CH}$, thiazole $), 44.21\left(\mathrm{CH}_{3}\right.$, pyridine ring), 45.60 ( $\mathrm{CH}$, pyrimidine), 51.34, 53.43 ( $2 \mathrm{CH}_{2}$-pyridine), $124.24(\mathrm{CH}$, methylene $), 126.43$, $126.82,127.10,128.13,128.52,128.73,135.21,143.15$ (12C, Ar-C), 136.70 (C-pyridine), 129.23, 134.64, 163.41 (3C-pyrimidine), $176.2(C=\mathrm{O}) ; \mathrm{MS}(\mathrm{EI}): \mathrm{m} / \mathrm{z}$ $470\left[\mathrm{M}^{+}\right](91), 472\left(\mathrm{M}^{+}+2\right)(60), 474\left(\mathrm{M}^{+}+4\right)(31), 468$ $\left(\mathrm{M}^{+}-\mathrm{H}_{2}\right)$ (100), $434\left(\mathrm{M}^{+}-\mathrm{Cl}\right)$ (18); Anal. Calcd. for $\mathrm{C}_{24} \mathrm{H}_{21} \mathrm{~N}_{3} \mathrm{SOCl}_{2}$ : C, 61.27; H, 4.50; N, 8.93; S,6.81. Found: C, 61.29; H, 4.52; N, 8.91; S,6.78.

9-(4-Chlorobenzylidene)-6,7,8,9-tetrahydro-2,7dimethyl-5-(4-chlorophenyl)-2H-thiazolo-[3,2-a]pyrido $[4,3-d]$ pyrimidine-3(5H)-one $(5 \mathrm{~d})$ : Yield $62 \%$; mp 119-121 ${ }^{\circ} \mathrm{C}$; IR (KBr) cm ${ }^{-1}$ : $1726(\mathrm{C}=\mathrm{O}) ;{ }^{1} \mathrm{H}$ NMR (DMSO- $\left.\mathrm{d}_{6}\right): 1.43\left(\mathrm{~d}, 3 \mathrm{H}, \mathrm{CH}_{3}\right.$, thiazole ring), 2.14 (s, $\left.2 \mathrm{H}-\mathrm{C}_{6}\right), 2.68$ (s, $3 \mathrm{H}, \mathrm{CH}_{3}$, pyridine ring), 3.62-4.21 (2d, $\left.2 \mathrm{H}-\mathrm{C}_{8}\right), 4.50(\mathrm{q}, 1 \mathrm{H}-\mathrm{C}-2), 5.82\left(\mathrm{~s}, 1 \mathrm{H}-\mathrm{C}_{5}\right), 7.33-7.61(\mathrm{~m}$, 9H, Ar-H+ $\mathrm{CH}=\mathrm{C}) ;{ }^{13} \mathrm{C}$ NMR: $20.23\left(\mathrm{CH}_{3}\right.$, thiazole ring), 43.72-( $\mathrm{CH}$, thiazole), $44.23\left(\mathrm{CH}_{3}\right.$, pyridine ring), $45.61(\mathrm{CH}$, pyrimidine $), 51.28,53.42\left(2 \mathrm{CH}_{2}\right.$-pyridine $)$, 124.21 (CH, methylene), 126.44, 126.85, 127.13, $128.15,128.53,128.75,135.23,143.16$ (12C, Ar-C), 136.72 (C-pyridine), 129.25, 134.64, 163.43 (3Cpyrimidine), $176.5(C=\mathrm{O})$; MS $(\mathrm{EI}): \mathrm{m} / \mathrm{z} 470\left[\mathrm{M}^{+}\right](66)$, $472\left(\mathrm{M}^{+}+2\right)(41), 474\left(\mathrm{M}^{+}+4\right)(20), 469\left(\mathrm{M}^{+}-\mathrm{H}^{+}\right)(100)$, $357 \quad\left(\mathrm{M}^{+}-\mathrm{Cl}-\mathrm{C}_{6} \mathrm{H}_{4}\right)$ (12); Anal. Calcd. for $\mathrm{C}_{24} \mathrm{H}_{21} \mathrm{~N}_{3} \mathrm{SOCl}_{2}$ : C, 61.27; H, 4.50; N, 8.93; S,6.81. Found: C, 61.25; H, 4.48; N, 8.95; S,6.83.

\section{9-(4-Bromobenzylidene)-6,7,8,9-tetrahydro-2,7-} dimethyl-5-(4-bromophenyl)-2H-thiazolo[3,2a]pyrido[4,3-d $]$ pyrimidine-3(5H)-one $\quad(5 e)$ : Yield 61\%; mp 129-131 ${ }^{\circ} \mathrm{C}$; IR (KBr) cm ${ }^{-1}$ : $1725(\mathrm{C}=\mathrm{O}) ;{ }^{1} \mathrm{H}$ NMR (DMSO-d $)$ ): $1.41\left(\mathrm{~d}, 3 \mathrm{H}, \mathrm{CH}_{3}\right.$, thiazole ring), 2.12 (s, $\left.2 \mathrm{H}-\mathrm{C}_{6}\right), 2.67$ (s, 3H, $\mathrm{CH}_{3}$, pyridine ring), 3.60$4.20\left(2 \mathrm{~d}, 2 \mathrm{H}-\mathrm{C}_{8}\right), 4.51$ (q, $\left.1 \mathrm{H}-\mathrm{C}_{-2}\right), 5.80\left(\mathrm{~s}, 1 \mathrm{H}-\mathrm{C}_{5}\right)$, 7.31-7.60 (m, 9H, Ar-H+ $\mathrm{CH}=\mathrm{C}) ;{ }^{13} \mathrm{C}$ NMR: 20.20 $\left(\mathrm{CH}_{3}\right.$, thiazole ring), $43.70(\mathrm{CH}$, thiazole $), 44.21\left(\mathrm{CH}_{3}\right.$, pyridine ring), $45.60(\mathrm{CH}$, pyrimidine), 51.30, 53.40 (2 $\mathrm{CH}_{2}$-pyridine), 124.21 ( $\mathrm{CH}$, methylene), 126.39, $126.82,127.10,128.12,128.51,128.72,135.22,143.15$ (12C, Ar-C), 136.71 (C-pyridine), 129.22, 134.62, 163.41 (3C-pyrimidine), $176.52(C=\mathrm{O}) ; \mathrm{MS}(\mathrm{EI}): \mathrm{m} / \mathrm{z}$ $559\left[\mathrm{M}^{+}\right](29), 547\left(\mathrm{M}^{+}+2\right)(60), 549\left(\mathrm{M}^{+}+4\right)(27), 471$ [M+-S-CH (C=O)-CH $\mathrm{CH}_{3}$ (8), 43 (100); Anal. Calcd. for $\mathrm{C}_{24} \mathrm{H}_{21} \mathrm{~N}_{3} \mathrm{SOBr}_{2}$ : C, 51.53; H, 3.78; N, 7.51; S, 5.73. Found: C, 51.55; H, 3.81; N, 7.49; S,5.72. 
Synthesis of 5-aryl-2,9-diarylmethylene-6,7,8,9tetrahydro-7-methylthiazolo[3,2-a]pyrido-[4,3d]pyrimidin-3(5H)-ones (6a-e): Method A. A mixture of compounds $2 \mathrm{a}, \mathrm{b}, \mathrm{d}(0.05$ mole $)$, chloroacetic acid $(1.0 \mathrm{~g}, 0.01 \mathrm{~mole}), 2 \mathrm{~g}$ of fused sodium acetate in $20 \mathrm{~mL}$ of glacial acetic acid and $10 \mathrm{ml}$ of acetic anhydride was refluxed for $12 \mathrm{~min}$, then equimolecular amount of the appropriate aldehydes was added. The reaction mixture was refluxed for $2 \mathrm{~h}$ and then poured onto cold water, The solid formed was filtered off and crystallized from the proper solvent.

Method B. A mixture of $4 \mathrm{a}, \mathrm{b}, \mathrm{d}$ (0.01 mole), equimolecular amount of appropriate aldehyde and 30 $\mathrm{ml}$ of acetic anhydride was refluxed for $1 \mathrm{~h}$, left to cool, poured onto cold water. The solid formed was filtered off and crystallized from the proper solvent to give $6 \mathrm{a}-\mathrm{e}$. The products were identified by their $\mathrm{mp}$ and $R_{\mathrm{f}}$-values in comparison with authentic samples previously obtained by method A. Method A gave better yield than method B.

\section{2,9-Dibezylidene-5-phenyl-6,7,8,9-tetrahydro-7-} methylthiazolo[3,2-a]pyrido[4,3-d]pyrimidin-3(5H)one (6a): Yield $81 \%$ [A], $76 \%$ [B]; mp $208-210^{\circ} \mathrm{C}$; IR $(\mathrm{KBr}) \mathrm{cm}^{-1}: 1709(\mathrm{C}=\mathrm{O}) ;{ }^{1} \mathrm{H}$ NMR $\left(\mathrm{CDCl}_{3}\right): 2.31$ (s, $\left.3 \mathrm{H}, \mathrm{CH}_{3}\right), 3.11\left(\mathrm{~s}, 2 \mathrm{H}-\mathrm{C}_{6}\right), 3.42-3.81\left(2 \mathrm{~d}, 2 \mathrm{H}-\mathrm{C}_{8}\right), 5.50$ $\left(\mathrm{s}, 1 \mathrm{H}-\mathrm{C}_{5}\right), 7.30-7.60(\mathrm{~m}, 17 \mathrm{H}, \mathrm{Ar}-\mathrm{H}+2 \mathrm{CH}=\mathrm{C}) ;{ }^{13} \mathrm{C}$ NMR: $44.22\left(\mathrm{CH}_{3}\right), 45.62(\mathrm{CH}$, pyrimidine $), 51.28$, $53.42\left(2 \mathrm{CH}_{2}\right.$-pyridine $), 118$ (C, thiazole $), 124.30$, 142.22 (2CH, methylene), 126.42, 126.81, 127.30, $128.11,128.63,128.73,135.32,143.22$ (18C, Ar-C), 134.80 (C-pyridine), 129.61, 136.93, 163.20 (3Cpyrimidine), $166.61(C=\mathrm{O}) ; \mathrm{MS}(\mathrm{EI}): \mathrm{m} / \mathrm{z} 475\left[\mathrm{M}^{+}\right]$ (100), $474\left(\mathrm{M}^{+}-\mathrm{H}^{+}\right)$(95), $397\left(\mathrm{M}^{+}-\mathrm{C}_{6} \mathrm{H}_{5}\right)$ (59); Anal. Calcd. for $\mathrm{C}_{30} \mathrm{H}_{25} \mathrm{~N}_{3} \mathrm{SO}$ : C, 75.76; H, 5.29; N, 8.83; S,6.74. Found: C, 75.74; H, 5.31; N, 8.80; S,6.76.

2-(4-Chlorobenzylidene)-9-bezylidene-5-phenyl6,7,8,9-tetrahydro-7-methylthiazolo[3,2-

a]pyrido[4,3-d]pyrimidin-3(5H)-one (6b): Yield $89 \%$ [A], 66\% [B]; mp 151-153 ${ }^{\circ} \mathrm{C}$; IR (KBr) cm $\mathrm{cm}^{-1}: 1719$ $(\mathrm{C}=\mathrm{O}) ;{ }^{1} \mathrm{H}$ NMR $\left(\mathrm{CDCl}_{3}\right): 2.41\left(\mathrm{~s}, 3 \mathrm{H}, \mathrm{CH}_{3}\right), 2.60-3.11$ $\left(\mathrm{d}, 2 \mathrm{H}-\mathrm{C}_{6}\right), 3.22-3.41\left(2 \mathrm{~d}, 2 \mathrm{H}-\mathrm{C}_{8}\right), 6.10\left(\mathrm{~s}, 1 \mathrm{H}-\mathrm{C}_{5}\right)$, 7.30-7.62 (m, 14H, Ar-H ), $7.71\left(\mathrm{~s}, 1 \mathrm{H}, \mathrm{CH}=\mathrm{C}_{9}\right), 7.89$ ( s, $\left.1 \mathrm{H}, \mathrm{CH}=\mathrm{C}_{2}\right) ;{ }^{13} \mathrm{C}$ NMR: $44.25\left(\mathrm{CH}_{3}\right), 45.65(\mathrm{CH}$, pyrimidine $), 51.35,53.45\left(2 \mathrm{CH}_{2}\right.$-pyridine $), 118.20(\mathrm{C}$, thiazole), $124.60,142.23(2 \mathrm{CH}$, methylene), 126.45, $126.84,127.33,128.14,128.66,128.76,135.35,143.25$ (18C, Ar-C), 134.70 (C-pyridine), 129.64, 136.90, 163.23 (3C-pyrimidine), $166.63(C=\mathrm{O}) ; \mathrm{MS}(\mathrm{EI}): \mathrm{m} / \mathrm{z}$ $510\left[\mathrm{M}^{+}\right](39), 512\left(\mathrm{M}^{+}+2\right)(63), 509\left(\mathrm{M}^{+}-\mathrm{H}^{+}\right)(100)$, $432\left(509-\mathrm{C}_{6} \mathrm{H}_{5}\right)$; Anal. Calcd. for $\mathrm{C}_{30} \mathrm{H}_{24} \mathrm{~N}_{3} \mathrm{SOCl}$ : C, 70.64; H, 4.74; N, 8.24; S, 6.28. Found: C, 70.62; H, $4.72 ; \mathrm{N}, 8.26 ; \mathrm{S}, 6.26$.
2-(4-Flourodibezylidene)-9-(4-chlorobenzylidene)-5(4-chlorophenyl)-6,7,8,9-tetrahydro-7methylthiazolo[3,2-a]pyrido[4,3-d]pyrimidin-3(5H)one (6c): Yield $85 \%$ [A], $68 \%$ [B]; mp $182-185{ }^{\circ} \mathrm{C}$; IR $(\mathrm{KBr}) \mathrm{cm}^{-1}: 1721(\mathrm{C}=\mathrm{O}) ;{ }^{1} \mathrm{H}$ NMR $\left(\mathrm{CDCl}_{3}\right): 2.45(\mathrm{~s}$, $\left.3 \mathrm{H}, \mathrm{CH}_{3}\right), 2.61-3.13\left(\mathrm{~d}, 2 \mathrm{H}-\mathrm{C}_{6}\right), 3.19-3.83(2 \mathrm{~d}, 2 \mathrm{H}-$ $\left.\mathrm{C}_{8}\right), 5.60\left(\mathrm{~s}, 1 \mathrm{H}-\mathrm{C}_{5}\right), 7.10-7.60(\mathrm{~m}, 12 \mathrm{H}, \mathrm{Ar}-\mathrm{H}), 7.76($ $\left.\mathrm{s}, 1 \mathrm{H}, \mathrm{CH}=\mathrm{C}_{9}\right), 7.91\left(\mathrm{~s}, 1 \mathrm{H}, \mathrm{CH}=\mathrm{C}_{2}\right) ;{ }^{13} \mathrm{C} \mathrm{NMR}$ : $44.23\left(\mathrm{CH}_{3}\right), 45.64(\mathrm{CH}$, pyrimidine $), 51.34,53.43$ (2 $\mathrm{CH}_{2}$-pyridine), 118.10 (C, thiazole), 124.62, 142.21 (2CH, methylene), 126.40, 126.80, 127.30, 128.12, 128.64, 128.75, 135.33, 143.24 (18C, Ar-C), 134.69 (C-pyridine), 129.58, 136.91, 163.20 (3C-pyrimidine), $166.60(C=\mathrm{O}) ; \mathrm{MS}(\mathrm{EI}): \mathrm{m} / \mathrm{z} 562\left[\mathrm{M}^{+}\right](90), 564$ $\left(\mathrm{M}^{+}+2\right)(59), 566\left(\mathrm{M}^{+}+4\right)(33), 467 \quad\left(\mathrm{M}^{+}-\mathrm{F}-\mathrm{C}_{6} \mathrm{H}_{4}\right)$ (100); Anal. Calcd. for $\mathrm{C}_{30} \mathrm{H}_{22} \mathrm{~N}_{3} \mathrm{SOCl}_{2} \mathrm{~F}$ : C, 64.05; H, 3.94; N, 7.47; S, 5.70. Found: C, 64.07; H, 3.96; N, 7.49; S, 5.67.

2-(4-Chlorodibezylidene)-9-(4-flourobenzylidene)-5(4-flourrophenyl)-6,7,8,9-tetrahydro-7methylthiazolo[3,2-a]pyrido[4,3-d]pyrimidin-3(5H)one (6d): Yield $85 \%$ [A], 75\% [B]; mp $155-157^{\circ} \mathrm{C}$; IR $(\mathrm{KBr}) \mathrm{cm}^{-1}: 1723(\mathrm{C}=\mathrm{O}) ;{ }^{1} \mathrm{H}$ NMR $\left(\mathrm{CDCl}_{3}\right): 2.44$ (s, 3H, $\left.\mathrm{CH}_{3}\right), 2.62-3.15$ (d, $\left.2 \mathrm{H}-\mathrm{C}_{6}\right), 3.21-3.84\left(2 \mathrm{~d}, 2 \mathrm{H}-\mathrm{C}_{8}\right), 5.62$ $\left(\mathrm{s}, 1 \mathrm{H}-\mathrm{C}_{5}\right), 7.11-7.61(\mathrm{~m}, 12 \mathrm{H}, \mathrm{Ar}-\mathrm{H}), 7.70(\mathrm{~s}, 1 \mathrm{H}$, $\left.\mathrm{CH}=\mathrm{C}_{9}\right), 7.82\left(\mathrm{~s}, 1 \mathrm{H}, \mathrm{CH}=\mathrm{C}_{2}\right) ;{ }^{13} \mathrm{C}$ NMR: $44.20\left(\mathrm{CH}_{3}\right)$, $45.61(\mathrm{CH}$, pyrimidine $), 51.30,53.41\left(2 \mathrm{CH}_{2}\right.$-pyridine $)$, 118.00 (C, thiazole), 124.63, 142.22 (2CH, methylene), 126.44, 126.83, 127.33, 128.13, 128.65, 128.75, 135.35, 143.25 (18C, Ar-C), 134.68 (C-pyridine), 129.59, 136.92, 163.21 (3C-pyrimidine), $166.61(C=\mathrm{O})$; MS (EI): $\mathrm{m} / \mathrm{z} 545\left[\mathrm{M}^{+}\right]$(71), $547\left(\mathrm{M}^{+}+2\right)(27), 544\left(\mathrm{M}^{+}-\mathrm{H}^{+}\right)(100)$, 433 (544-Cl- $\left.\mathrm{C}_{6} \mathrm{H}_{4}\right)$ (85); Anal. Calcd. for $\mathrm{C}_{30} \mathrm{H}_{22} \mathrm{~N}_{3} \mathrm{SOClF}_{2}$ : C, 65.99; H, 4.06; N, 7.69; S, 5.87. Found: C, 65.97; H, 4.04; N, 7.68; S, 5.89.

2-(4-Methoxydibezylidene)-9-(4-chlorobenzylidene)5-(4-chlorophenyl)-6,7,8,9-tetrahydro-7methylthiazolo[3,2-a]pyrido[4,3-d]pyrimidin-3(5H)one (6e): Yield $72 \%$ [A], $65 \%$ [B]; mp $162-164^{\circ} \mathrm{C}$; IR $(\mathrm{KBr}) \mathrm{cm}^{-1}: 1711(\mathrm{C}=\mathrm{O}) ;{ }^{1} \mathrm{H}$ NMR $\left(\mathrm{CDCl}_{3}\right): 2.25$ (s, $\left.3 \mathrm{H}, \mathrm{CH}_{3}\right), 2.90\left(\mathrm{~s}, 3 \mathrm{H}, \mathrm{OCH}_{3}\right), 3.11-3.36\left(\mathrm{~d}, 2 \mathrm{H}-\mathrm{C}_{6}\right)$, 3.51-3.81 (2d, 2H-C $), 5.91\left(\mathrm{~s}, 1 \mathrm{H}-\mathrm{C}_{5}\right), 7.11-7.63(\mathrm{~m}$, $14 \mathrm{H}, \mathrm{Ar}-\mathrm{H}+2 \mathrm{CH}=\mathrm{C}) ;{ }^{13} \mathrm{C} \mathrm{NMR}: 44.19\left(\mathrm{CH}_{3}\right), 45.58$ (CH, pyrimidine), 51.37, $53.38\left(2 \mathrm{CH}_{2}\right.$-pyridine), 56.01 $\left(\mathrm{OCH}_{3}\right), 118.20(\mathrm{C}$, thiazole $), 124.50,142.20(2 \mathrm{CH}$, methylene), 126.40, 126.80, 127.30, 128.10, 128.60, $128.70,135.30,143.20$ (18C, Ar-C), 134.60 (Cpyridine), 129.61, 136.90, 163.20 (3C-pyrimidine), $166.60(C=\mathrm{O})$; MS $(\mathrm{EI}): \mathrm{m} / \mathrm{z} 574\left[\mathrm{M}^{+}\right](86), 576$ $\left(\mathrm{M}^{+}+2\right)(57), 578\left(\mathrm{M}^{+}+4\right)(31), 543\left(\mathrm{M}^{+}-\mathrm{OCH}_{3}\right)(60)$, 
$467 \quad\left(\mathrm{M}^{+}-\mathrm{OCH}_{3} \mathrm{C}_{6} \mathrm{H}_{4}\right)$ (100); Anal. Calcd. for $\mathrm{C}_{31} \mathrm{H}_{25} \mathrm{~N}_{3} \mathrm{SO}_{2} \mathrm{Cl}_{2}$ : C, 64.80; H, 4.38; N, 7.31; S, 5.58. Found: C, 64.78; H, 4.36; N, 7.34; S, 5.59.

\section{Synthesis of 5-Aryl-9-arylmethylene-6,7,8,9- tetrahydro-7-methyl-2-arylyldiazenyl-2H- thiazolo[3,2-a]pyrido[4,3-d]pyrimidin-3(5H)-ones}

(7a-d): The aromatic amine, namely, aniline, ptoluidine or $\mathrm{p}$-anisidine (0.01 mole) was dissolved in 3 $\mathrm{ml} \mathrm{HCl}(70 \%)$ and cooled to $0^{\circ} \mathrm{C}$, then treated with a solution of $0.7 \mathrm{~g}$ sodium nitrite in $2 \mathrm{ml}$ water, the diazonium salt was cooled for $15 \mathrm{~min}$, added gradually with stirring to cooled solution of compound $4 \mathrm{a}, \mathrm{d}$ (0.01 mole) in $10 \mathrm{~mL}$ of pyridine, the reaction mixture was cooled for $30 \mathrm{~min}$ and poured onto $100 \mathrm{~mL}$ water. The solid formed was crystallized from the proper solvent to give compounds $7 \mathrm{a}$-d.

\section{9-Benzylidene-6,7,8,9-tetrahydro-5-phenyl-7- methyl-2-(4-tolyldiazenyl)-2H-thiazolo[3,2-} a]pyrido[4,3-d]pyrimidin-3(5H)-one (7a): Yield 79\%; mp 215-217 ${ }^{\circ} \mathrm{C}$; IR $(\mathrm{KBr}) \mathrm{cm}^{-1}$ : $1728(\mathrm{C}=\mathrm{O}) ;{ }^{1} \mathrm{H}$ NMR $\left(\mathrm{CDCl}_{3}\right): 2.28\left(\mathrm{~s}, 3 \mathrm{H}, \mathrm{CH}_{3}-\mathrm{N}\right), 2.34\left(\mathrm{~s}, 3 \mathrm{H}, \mathrm{CH}_{3}-\mathrm{Ph}\right)$, 2.82-3.15 (d, 2H-C 6 ), 3.51-3.84 (m, 2H-C $), 4.10(\mathrm{~s}$, $\left.1 \mathrm{H}, \mathrm{CH}=\mathrm{C}_{2}\right), 5.72\left(\mathrm{~s}, 1 \mathrm{H}-\mathrm{C}_{5}\right), 6.60\left(\mathrm{~s}, 1 \mathrm{H}+\mathrm{CH}=\mathrm{C}_{9}\right)$, 7.21-7.61 (m, 14H, Ar-H); ${ }^{13} \mathrm{C}$ NMR: $24.32\left(\mathrm{CH}_{3}-\mathrm{Ph}\right)$, $44.22\left(\mathrm{CH}_{3}\right), 45.41(\mathrm{CH}$, pyrimidine $), 53.42,64.60$ (2 $\mathrm{CH}_{2}$-pyridine), $64.00(\mathrm{CH}$, thiazole $), 124.23(\mathrm{CH}$, methylene), 126.24, 126.73, 127.13, 128.13, 128.63, 128.74, 135.26, 143.22 (18C, Ar-C), 136.67 (Cpyridine), 129.18, 136.90, 163.23 (3C-pyrimidine), $170.61(\mathrm{C}=\mathrm{O})$; MS (EI): m/z $505\left[\mathrm{M}^{+}\right](22), 386\left(\mathrm{M}^{+}-\right.$ $\left.\mathrm{CH}_{3}-\mathrm{C}_{6} \mathrm{H}_{5}-\mathrm{N}=\mathrm{N}\right)$ (100); Anal. Calcd. for $\mathrm{C}_{30} \mathrm{H}_{27} \mathrm{~N}_{5} \mathrm{OS}$ : C, 71.25; H, 5.38; N, 13.85; S, 6.34. Found: C, 71.23; H, 5.40; N, 13.82; S, 6.36 .

9-(4-Chlorobenzylidene)-6,7,8,9-tetrahydro-5-(4chlorophenyl)-7-methyl-2(2-phenyldiazenyl)-2Hthiazolo[3,2-a]pyrido[4,3-d]pyrimidin-3(5H)-one (7b): Yield 78\%; mp 211-213 ${ }^{\circ} \mathrm{C}$; IR (KBr) $\mathrm{cm}^{-1}$ : 1726 $(\mathrm{C}=\mathrm{O}) ;{ }^{1} \mathrm{H} \mathrm{NMR}\left(\mathrm{CDCl}_{3}\right): 2.49\left(\mathrm{~s}, 3 \mathrm{H}, \mathrm{CH}_{3}\right), 2.81-3.20$ $\left(\mathrm{d}, 2 \mathrm{H}-\mathrm{C}_{6}\right), 3.50-3.79\left(\mathrm{~m}, 2 \mathrm{H}-\mathrm{C}_{8}\right), 4.20(\mathrm{~s}, 1 \mathrm{H}$, $\left.\mathrm{CH}=\mathrm{C}_{2}\right), 5.82\left(\mathrm{~s}, 1 \mathrm{H}-\mathrm{C}_{5}\right), 6.63\left(\mathrm{~s}, 1 \mathrm{H},+\mathrm{CH}=\mathrm{C}_{9}\right), 7.28-$ 7.66 (m, 13H, Ar-H); ${ }^{13} \mathrm{C}$ NMR: $44.20\left(\mathrm{CH}_{3}\right), 45.40$ (CH, pyrimidine), 51.40, $53.40\left(2 \mathrm{CH}_{2}\right.$-pyridine $), 64.50$ $(\mathrm{CH}$, thiazole $), 124.00(\mathrm{CH}$, methylene), 126.20, $126.70,127.10,128.03,128.60,128.70,135.20,143.20$ (18C, Ar-C), 136.64 (C-pyridine), 129.09, 134.91, 163.00 (3C-pyrimidine), $171.61(\mathrm{C}=\mathrm{O}) ; \mathrm{MS}(\mathrm{EI}): \mathrm{m} / \mathrm{z}$ 560[M $\left.\mathrm{M}^{+}\right](92), 562\left(\mathrm{M}^{+}+2\right)(60), 564\left(\mathrm{M}^{+}+4\right)(35), 455$ $\left(\mathrm{M}^{+}-\mathrm{C}_{6} \mathrm{H}_{5}-\mathrm{N}=\mathrm{N}\right) \quad$ (100); Anal. Calcd. for $\mathrm{C}_{29} \mathrm{H}_{23} \mathrm{~N}_{5} \mathrm{OSCl}_{2}$ : C, 62.14; H, 4.13; N, 12.49; S, 5.72. Found: C, 62.11; H, 4.11; N, 12.51; S, 5.70.
9-(4-Chlorobenzylidene)-6,7,8,9-tetrahydro-5-(4chlorophenyl)-7-methyl-2(4-tolyl-diazenyl)-2Hthiazolo[3,2-a]pyrido[4,3-d]pyrimidin-3(5H)-one (7c): Yield 77\%; mp 202-205 ${ }^{\circ}$; IR $(\mathrm{KBr}) \mathrm{cm}^{-1}$ : $1730(\mathrm{C}=\mathrm{O}) ;{ }^{1} \mathrm{H}$ NMR $\left(\mathrm{CDCl}_{3}\right): 2.28\left(\mathrm{~s}, 3 \mathrm{H}, \mathrm{CH}_{3}-\mathrm{N}\right)$, 2.35 (s, $\left.3 \mathrm{H}, \mathrm{CH}_{3}-\mathrm{Ph}\right), 2.83-3.25\left(\mathrm{~d}, 2 \mathrm{H}-\mathrm{C}_{6}\right), 3.52-3.85$ $\left(\mathrm{m}, 2 \mathrm{H}-\mathrm{C}_{8}\right), 4.10\left(\mathrm{~s}, 1 \mathrm{H}, \mathrm{CH}=\mathrm{C}_{2}\right), 5.82\left(\mathrm{~s}, 1 \mathrm{H}-\mathrm{C}_{5}\right), 6.60$ $\left(\mathrm{s}, 1 \mathrm{H}+\mathrm{CH}=\mathrm{C}_{9}\right), 7.23-7.81(\mathrm{~m}, 12 \mathrm{H}, \mathrm{Ar}-\mathrm{H}) ;{ }^{13} \mathrm{C}$ NMR: $24.31\left(\mathrm{CH}_{3}-\mathrm{Ph}\right), 44.23\left(\mathrm{CH}_{3}\right), 45.40(\mathrm{CH}$, pyrimidine $)$, 51.43, $53.43\left(2 \mathrm{CH}_{2}\right.$-pyridine $), 64.20(\mathrm{CH}$, thiazole $)$, 124.10 (CH, methylene), 126.24, 126.73, 127.13, 128.14, 128.63, 128.70, 135.24, 143.21 (18C, Ar-C), 136.66 (C-pyridine), 129.05, 134.88, 163.22 (3Cpyrimidine), $171.51(C=\mathrm{O}) ; \mathrm{MS}(\mathrm{EI}): \mathrm{m} / \mathrm{z} 574\left[\mathrm{M}^{+}\right]$ (79), $576\left(\mathrm{M}^{+}+2\right)(41), 578\left(\mathrm{M}^{+}+4\right)(25), 483\left(\mathrm{M}^{+}-\right.$ $\mathrm{CH}_{3} \mathrm{C}_{6} \mathrm{H}_{4}$ ) (100); Anal. Calcd. for $\mathrm{C}_{30} \mathrm{H}_{25} \mathrm{~N}_{5} \mathrm{OSCl}_{2}$ : C, 62.71; H, 4.38; N, 12.19; S, 5.58. Found: C, 62.73; H, $4.40 ; \mathrm{N}, 12.20 ; \mathrm{S}, 5.56$.

9-(4-Chlorobenzylidene)-6,7,8,9-tetrahydro-5-(4chlorophenyl)-7-methyl-2(4-methoxyphenyldiazenyl)-2H-thiazolo[3,2-a]pyrido[4,3-d]pyrimidin3(5H)-one (7d): Yield 80\%; mp 198-201 ${ }^{\circ} \mathrm{C}$; IR (KBr) $\mathrm{cm}^{-1}: 1710(\mathrm{C}=\mathrm{O}) ;{ }^{1} \mathrm{H}$ NMR (DMSO-d 6$): 2.28(\mathrm{~s}, 3 \mathrm{H}$, $\mathrm{CH}_{3}-\mathrm{N}$ ), 2.83-3.15 (d, 2H-C $), 3.86$ (s, 3H, $\left.\mathrm{OCH}_{3}-\mathrm{Ph}\right)$, 3.51-3.95 (m, 2H-C $\left.\mathrm{C}_{8}\right), 4.10\left(\mathrm{~s}, 1 \mathrm{H}, \mathrm{CH}=\mathrm{C}_{2}\right), 5.72(\mathrm{~s}$, $\left.1 \mathrm{H}-\mathrm{C}_{5}\right), 7.15-7.81\left(\mathrm{~m}, 15 \mathrm{H}, \mathrm{Ar}-\mathrm{H}+\mathrm{CH}=\mathrm{C}_{9}\right) ;{ }^{13} \mathrm{C} \mathrm{NMR}$ : $44.23\left(\mathrm{CH}_{3}\right), 45.43(\mathrm{CH}$, pyrimidine $), 51.42,53.41$ (2 $\mathrm{CH}_{2}$-pyridine), $57.10 \quad\left(\mathrm{OCH}_{3}-\mathrm{Ph}\right), 64.62 \quad(\mathrm{CH}$, thiazole), 124.11 ( $\mathrm{CH}$, methylene), 126.24, 126.73, 127.13, 128.13, 128.63, 128.74, 135.44, 143.31 (18C, Ar-C), 136.68 (C-pyridine), 128.54, 134.93, 163.24 (3C-pyrimidine), $170.55(\mathrm{C}=\mathrm{O})$; MS (EI): m/z $590\left[\mathrm{M}^{+}\right]$ (79), $592\left(\mathrm{M}^{+}+2\right)(41), 594\left(\mathrm{M}^{+}+4\right)(25), 479\left(\mathrm{M}^{+}-\right.$ $\mathrm{ClC}_{6} \mathrm{H}_{4}$ ) (100); Anal. Calcd. for $\mathrm{C}_{30} \mathrm{H}_{25} \mathrm{~N}_{5} \mathrm{O}_{2} \mathrm{SCl}_{2}$ : C, 61.01; H, 4.26; N, 11.86; S, 5.43. Found: C, 61.03; H, $4.24 ; \mathrm{N}, 11.88 ; \mathrm{S}, 5.41$.

Anticancer activity: Some of the synthesized compounds were selected and screened for their anticancer activity. Each compound was tested at five different concentrations against 60 cell lines of nine types of human cancers, namely, leukemia, lung, colon, CNS, melanoma, ovarian, renal, prostate and breast cancer. Results are expressed as $\log _{10} \mathrm{GI}_{50}$, which the drug concentration (M) is causing a 50\% reduction in the net protein increase in control cells during the drug incubation (Negaoui et al., 2009) Table 1. Some of the synthesized compounds showed good anticancer activity at low concentration compared with 5-fluorodeoxyuridine $\log _{10} \mathrm{GI}_{50}=-4.7$ as $\begin{array}{lll} & \text { reference } & \\ \text { as } & & \end{array}$ 
Am. J. Biochem. \& Biotech., 7 (2): 43-54, 2011

Table 1: In vitro inhibition results of cancer cell lines of the tested derivatives (GI 50 (iM))*

\begin{tabular}{|c|c|c|c|c|c|c|c|c|c|c|c|}
\hline \multirow[b]{2}{*}{ Panel/cell line } & \multicolumn{11}{|c|}{ Compound } \\
\hline & $3 \mathrm{~b}$ & & $3 \mathrm{~d}$ & $4 \mathrm{a}$ & $4 \mathrm{~b}$ & $4 \mathrm{~d}$ & $5 \mathrm{~b}$ & $5 \mathrm{c}$ & $5 \mathrm{~d}$ & $6 \mathrm{~b}$ & $7 \mathrm{~b}$ \\
\hline \multicolumn{12}{|c|}{ Non-small cell lung cancer } \\
\hline A 549/ATCC & -5.3 & & -5.3 & -5.3 & -5.3 & -5.3 & -5.3 & -5.3 & -5.3 & -5.3 & -5.3 \\
\hline EKVX & -5.3 & & -5.3 & -5.3 & -5.3 & -5.3 & -5.3 & -5.3 & -5.3 & -5.3 & -5.3 \\
\hline HOP-62 & -5.3 & & -5.3 & -5.3 & -5.3 & -5.3 & -5.3 & -5.3 & -5.3 & -5.3 & -5.3 \\
\hline HOP-92 & -5.3 & & -5.3 & -5.3 & -5.3 & -5.3 & -5.3 & -5.3 & -5.3 & -5.3 & -5.3 \\
\hline NCI-H226 & -5.3 & & -5.3 & -5.3 & -5.3 & -5.3 & -5.3 & -5.3 & -5.3 & -5.3 & -5.3 \\
\hline NCI-H23 & -5.3 & & -5.3 & -5.3 & -5.3 & -5.3 & -5.3 & -5.3 & -5.3 & -5.3 & -5.3 \\
\hline NCI-H322M & -5.3 & & -5.3 & -5.3 & -5.3 & -5.3 & -5.3 & -5.3 & -5.3 & -5.3 & -5.3 \\
\hline NCI-H460 & -5.3 & & -5.3 & -5.3 & -5.3 & -5.3 & -5.3 & -5.3 & -5.3 & -5.3 & -5.3 \\
\hline NCI-H522 & -5.3 & & -5.3 & -5.3 & -5.3 & -5.3 & -5.3 & -5.3 & -5.3 & -5.3 & -5.3 \\
\hline \multicolumn{12}{|l|}{ Leukemia } \\
\hline CCRF-CE & -4.3 & & -4.3 & -4.3 & -4.3 & -4.3 & -4.3 & -4.3 & -4.3 & -4.3 & -4.3 \\
\hline CCRF-CEM & -4.3 & & -4.3 & -4.3 & -4.3 & -4.3 & -4.3 & -4.3 & -4.3 & -4.3 & -4.3 \\
\hline HL-60 (TB) & -4.3 & & -4.3 & -4.3 & -4.3 & -4.3 & -4.3 & -4.3 & -4.3 & -4.3 & -4.3 \\
\hline K-562 & -4.3 & & -4.3 & -4.3 & -4.3 & -4.3 & -4.3 & -4.3 & -4.3 & -4.3 & -4.3 \\
\hline MoLT-4 & -4.3 & & -4.3 & -4.3 & -4.3 & -4.3 & -4.3 & -4.3 & -4.3 & -4.3 & -4.3 \\
\hline RPMI-8226 & -4.3 & & -4.3 & -4.3 & -4.3 & -4.3 & -4.3 & -4.3 & -4.3 & -4.3 & -4.3 \\
\hline & -4.3 & & -4.3 & -4.3 & -4.3 & -4.3 & -4.3 & -4.3 & -4.3 & -4.3 & -4.3 \\
\hline \multicolumn{12}{|l|}{ CNS cancer } \\
\hline SF-268 & -4.3 & & -4.3 & -4.3 & -4.3 & -4.3 & -4.3 & -4.3 & -4.3 & -4.3 & -4.3 \\
\hline SF-295 & -4.3 & & -4.3 & -4.3 & -4.3 & -4.3 & -4.3 & -4.3 & -4.3 & -4.3 & -4.3 \\
\hline SNB-19 & -4.3 & & -4.3 & -4.3 & -4.3 & -4.3 & -4.3 & -4.3 & -4.3 & -4.3 & -4.3 \\
\hline SNB-75 & & -4.3 & -4.3 & -4.3 & -4.3 & -4.3 & -4.3 & -4.3 & -4.3 & -4.3 & -4.3 \\
\hline $\mathrm{U} 251$ & -4.3 & & -4.3 & -4.3 & -4.3 & -4.3 & -4.3 & -4.3 & -4.3 & -4.3 & -4.3 \\
\hline \multicolumn{12}{|l|}{ Colon Cancer } \\
\hline COLO 205 & -4.0 & & -4.3 & -4.3 & -4.3 & -4.3 & -4.3 & -4.0 & -4.3 & -4.3 & -4.3 \\
\hline HCC-2998 & -4.0 & & -4.3 & -4.3 & -4.3 & -4.3 & -4.3 & -4.0 & -4.3 & -4.3 & -4.3 \\
\hline НCT-116 & -4.0 & & -4.3 & -4.3 & -4.3 & -4.3 & -4.3 & -4.0 & -4.3 & -4.3 & -4.3 \\
\hline НCT-15 & & -4.0 & -4.3 & -4.3 & -4.3 & -4.3 & -4.3 & -4.0 & -4.3 & -4.3 & -4.3 \\
\hline HT29 & -4.0 & & -4.3 & -4.3 & -4.3 & -4.3 & -4.3 & -4.0 & -4.3 & -4.3 & -4.3 \\
\hline KM12 & -4.0 & & -4.3 & -4.3 & -4.3 & -4.3 & -4.3 & -4.0 & -4.3 & -4.3 & -4.3 \\
\hline \multirow[t]{2}{*}{ SW-620 } & & & -4.0 & -4.3 & -4.3 & -4.3 & -4.3 & -4.3 & -4.0 & -4.3 & -4.3 \\
\hline & -4.3 & & & & & & & & & & \\
\hline $\begin{array}{l}\text { Breast Cancer } \\
\text { MCF } 7\end{array}$ & -4.3 & & & & & & & & & & -4.3 \\
\hline NCI/ADR-RES & $\begin{array}{l}-4.3 \\
-4.3\end{array}$ & & $\begin{array}{l}-4.5 \\
-4.3\end{array}$ & $\begin{array}{l}-4.3 \\
-4.3\end{array}$ & -4.3 & $\begin{array}{l}-4.3 \\
-4.3\end{array}$ & $\begin{array}{l}-4.3 \\
-4.3\end{array}$ & $\begin{array}{l}-4.3 \\
-4.3\end{array}$ & $\begin{array}{l}-4.3 \\
-4.3\end{array}$ & $\begin{array}{l}-4.3 \\
-4.3\end{array}$ & $\begin{array}{l}-4.3 \\
-4.3\end{array}$ \\
\hline MDA-MB-231/ATCC & -4.3 & & -4.3 & -4.3 & -4.3 & -4.3 & -4.3 & -4.3 & -4.3 & -4.3 & -4.3 \\
\hline HS 578T & -4.3 & & -4.3 & -4.3 & -4.3 & -4.3 & -4.3 & -4.3 & -4.3 & -4.3 & -4.3 \\
\hline MDA-MB-435 & -4.3 & & -4.3 & -4.3 & -4.3 & -4.3 & -4.3 & -4.3 & -4.3 & -4.3 & -4.3 \\
\hline BT-549 & -4.3 & & -4.3 & -4.3 & -4.3 & -4.3 & -4.3 & -4.3 & -4.3 & -4.3 & -4.3 \\
\hline $\mathrm{T}-47 \mathrm{D}$ & -4.3 & & -4.3 & -4.3 & -4.3 & -4.3 & -4.3 & -4.3 & -4.3 & -4.3 & -4.3 \\
\hline Ovarian Cancer & & & & & & & & & & & \\
\hline IGROVI & -4.3 & & -4.0 & -4.0 & -4.0 & -4.3 & -4.3 & -4.0 & -4.0 & -4.3 & -4.3 \\
\hline OVCAR-3 & -4.3 & & -4.0 & -4.0 & -4.0 & -4.3 & -4.3 & -4.0 & -4.0 & -4.3 & -4.3 \\
\hline OVCAR-4 & & -4.3 & -4.0 & -4.0 & -4.0 & -4.3 & -4.3 & -4.0 .0 & -4.3 & -4.3 & -4.3 \\
\hline OVCAR-5 & -4.3 & & -4.0 & -4.0 & -4.0 & -4.3 & -4.3 & -4.0 & -4.0 & -4.3 & -4.3 \\
\hline OVCAR-8 & -4.3 & & -4.0 & -4.0 & -4.0 & -4.3 & -4.3 & -4.0 & -4.0 & -4.3 & -4.3 \\
\hline SK-OV-3 & -4.3 & & -4.0 & -4.0 & -4.0 & -4.3 & -4.3 & -4.0 & -4.0 & -4.3 & -4.3 \\
\hline Prostate Cancer & & & & & & & & & & & \\
\hline PC-3 & -4.3 & & -4.3 & -4.3 & -4.3 & -4.3 & -4.3 & -4.3 & -4.3 & -4.3 & -4.3 \\
\hline DU-145 & -4.3 & & -4.3 & -4.3 & -4.3 & -4.3 & -4.3 & -4.3 & -4.3 & -4.3 & -4.3 \\
\hline Renal Cancer & & & & & & & & & & & \\
\hline $786-0$ & -4.0 & & -4.3 & -4.3 & -4.3 & -4.3 & -4.3 & -4.0 & -4.3 & -4.3 & -4.3 \\
\hline A 498 & -4.0 & & -4.3 & -4.3 & -4.3 & -4.3 & -4.3 & -4.0 & -4.3 & -4.3 & -4.3 \\
\hline ACHN & -4.0 & & -4.3 & -4.3 & -4.3 & -4.3 & -4.3 & -4.0 & -4.3 & -4.3 & -4.3 \\
\hline CAKI-1 & -4.0 & & -4.3 & -4.3 & -4.3 & -4.3 & -4.3 & -4.0 & -4.3 & -4.3 & -4.3 \\
\hline RXF-393 & -4.0 & & -4.3 & -4.3 & -4.3 & -4.3 & -4.3 & -4.0 & -4.3 & -4.3 & -4.3 \\
\hline SN12C & -4.0 & & -4.3 & -4.3 & -4.3 & -4.3 & -4.3 & -4.0 & -4.3 & -4.3 & -4.3 \\
\hline TK-10 & -4.0 & & -4.3 & -4.3 & -4.3 & -4.3 & -4.3 & -4.0 & -4.3 & -4.3 & -4.3 \\
\hline UO-31 & -4.0 & & -4.3 & -4.3 & -4.3 & -4.3 & -4.3 & -4.0 & -4.3 & -4.3 & -4.3 \\
\hline Melanoma & & & & & & & & & & & \\
\hline LOXIMVI & -4.0 & & -4.3 & -4.3 & -4.3 & -4.3 & -4.3 & -4.0 & -4.3 & -4.3 & -4.3 \\
\hline MALME-3M & -4.0 & & -4.3 & -4.3 & -4.3 & -4.3 & -4.3 & -4.0 & -4.3 & -4.3 & -4.3 \\
\hline M 14 & -4.0 & & -4.3 & -4.3 & -4.3 & -4.3 & -4.3 & -4.0 & -4.3 & -4.3 & -4.3 \\
\hline SK-MEL-2 & -4.0 & & -4.3 & -4.3 & -4.3 & -4.3 & -4.3 & -4.0 & -4.3 & -4.3 & -4.3 \\
\hline SK-MEL-28 & -4.0 & & -4.3 & -4.3 & -4.3 & -4.3 & -4.3 & -4.0 & -4.3 & -4.3 & -4.3 \\
\hline SK-MEL-5 & -4.0 & & -4.3 & -4.3 & -4.3 & -4.3 & -4.3 & -4.0 & -4.3 & -4.3 & -4.3 \\
\hline UACC- 257 & -4.0 & & -4.3 & -4.3 & -4.3 & -4.3 & -4.3 & -4.0 & -4.3 & -4.3 & -4.3 \\
\hline UACC-62 & -4.0 & & -4.3 & -4.3 & -4.3 & -4.3 & -4.3 & -4.0 & -4.3 & -4.3 & -4.3 \\
\hline
\end{tabular}

*: Data obtained from NCI's in vitro disease-oriented tumor cell screen; $\mathrm{GI}_{50}$ : drug molar concentration causing $50 \%$ cell growth inhibition, $\mathrm{NA}=$ No Activity 


\section{RESULTS}

In continuation to our search for new heterocyclic chemistry based anticancer, the suggestion, synthesis, structure elucidation of some thiopyrimidine derivatives 2-7 were realized herein using 3,5-bisarylmethylene-1methyl-4-piperidone and 4-aryl-8-arylmethelene-6methylpyrido[4,3-d]pyrimidine-2(1H)thiones as a starting materials. Some of the synthesized compounds were selected and screened for their anticancer activity. Each compound was tested at five different concentrations against 60 cell lines of nine types of human cancers, namely, leukemia, lung, colon, CNS, melanoma, ovarian, renal, prostate and breast cancer. Some of the tested compounds were better exhibited in vitro antitumor activities at low concentration $\left(\log _{10} \mathrm{GI}_{50}=-4.7\right)$ against the used human tumor cell lines. From the in vitro observed data it has been noticed that, some of the synthesized compounds seem to be the most active prepared derivatives against all the tested cell lines.

\section{DISCUSSION}

Chemistry: The synthetic strategy to synthesize the target products 2-7 is depicted in Fig. 1 and 2 . Preparation of 3, 5-bisarylmethylene-1-methyl-4piperidone (1a-f) was established according to the reported procedure (Lyle et al., 1973; Mcelvain and Rorig, 1948; Pattaraporn and Tharapong, 2009). The corresponding pyrimidine thione derivatives (2a-e) were obtained from condensation of 1a-e with thiourea in ethanolic potassium hydroxide solution under reflux. When compounds 1a-d, f reacted with 2-amino-2thiazoline in butanol/DMSO mixture afforded compounds 3a-e in good yields. Reaction of compounds 2a-e with chloroacetic acid or with 2-bromopropionic acid in the presence of sodium acetate in acetic acid/acetic anhydride mixture gave thiazolopyrimidene derivatives 4a-e and 5a-e, respectively (Fig. 1). Compounds $4 \mathrm{a}, \mathrm{b}, \mathrm{d}$ contain an active methylene group, when these compounds reacted with aromatic aldehydes in the presence of acetic acid/acetic anhydride mixture, the corresponding arylmethylene thiazolopyrimidines derivatives 6a-e were obtained (Fig. 2). The products 6a-e could be also obtained from reaction of $2 a, b, d$ with chloroacetic acid, followed by treatment with aromatic aldehyde in the presence of sodium acetate in refluxing acetic acid/acetic anhydride mixture. Compounds $4 \mathrm{a}, \mathrm{b}, \mathrm{d}$ were coupled with aryldiazonium salts in the presence of pyridine to give arylazo-thiazolopyrimidines derivatives 7a-d (Fig. 2).

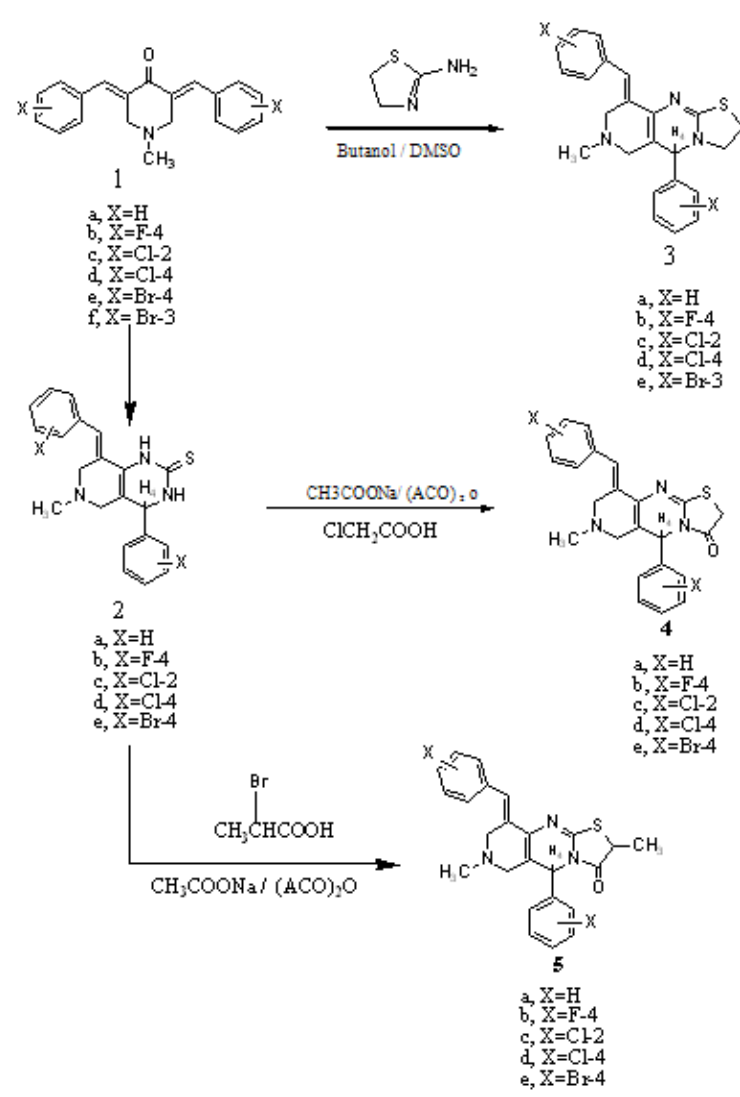

Fig. 1: Synthetic routes for compounds 1-5

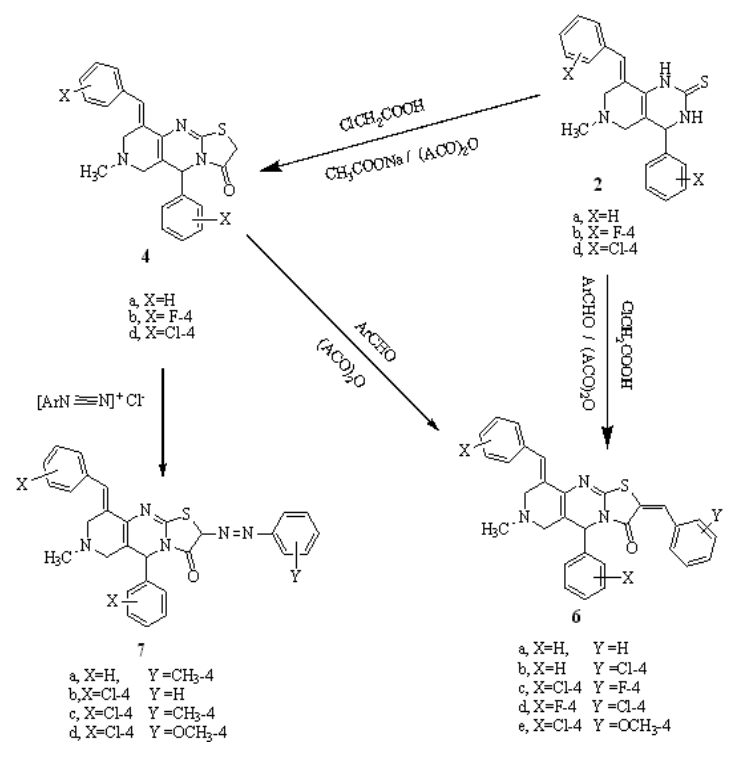

Fig. 2: Synthetic routes for compounds 6 and 7 
Antitumor screening: Antitumor activity screening for the synthesized compounds utilizing 59 different human tumor cell lines, representing leukemia, melanoma and cancers of the lung, colon, brain, ovary, breast, prostate as well as kidney, was carried out according to the previously reported standard procedure (Fylaktakidou et al., 2004; Jung et al., 2005; Ngoy et al., 2011 and Shuangning et al., 2010). The obtained results (Table 1) represent concentrations of the used investigated compounds resulting in growth inhibition of $50 \%\left(\mathrm{GI}_{50}\right)$ for the tested human tumor cell lines. From the in vitro observed data it has been noticed that, the selected compounds 3b, 3c, 3d, 4a, 4b, 4d, 5b, 5c, 5d, 6c, 6b, 6d, $7 \mathrm{~b}$ and $7 \mathrm{~d}$ seem to be the most active prepared derivatives against all the tested cell lines.

Structural-Activity Relationship (SAR): From the above-obtained results (Table 1), we can conclude that thiopyrimidine moieties fused to N-methylpipredine ring are essential for antitumor activities. In the present work, we can suggest that the anticancer activity is due to:

- The presence of nitrogen heterocyclic rings

- The most active compounds being 3b, 3c, 3d, 4a, $4 \mathrm{~b}, 4 \mathrm{~d}, 5 \mathrm{~b}, 5 \mathrm{c}, 5 \mathrm{~d}, 6 \mathrm{c}, 6 \mathrm{~b}, 6 \mathrm{~d}, 7 \mathrm{~b}$ and $7 \mathrm{~d}$ against all the tested cell lines.

- The presence of the nitrogen and sulfur atoms generally enhancing the activity

- The difference in activity between the compounds which is due to the indicated subsistents in the phenyl group of the molecule

\section{CONCLUSION}

In our previous works, we reported that fused pyrimidine derivatives were proved to be active anticancer agents. In the present work, a series of thiopyrimidine derivatives were synthesized using 3,5bisarylmethylene-1-methyl-4-piperidone and 4-aryl-8arylmethelene-6-methylpyrido[4,3-d]pyrimidine-

$2(1 H)$ thiones as a starting materials.

The antitumor activities of the newly synthesized compounds were evaluated utilizing 60 different human tumor cell lines, representing leukemia, melanoma, lung, colon, brain, ovary, breast, prostate as well as kidney. Some of the tested compounds were better exhibited in vitro antitumor activities at low concentration $\left(\log _{10} \mathrm{GI}_{50}\right.$ $=-4.7$ ) against the used human tumor cell lines. In the present work, we can suggest that the anticancer activity is due to the presence of nitrogen heterocyclic rings and the presence of the sulfur atom.

\section{ACKNOWLEDGEMENT}

The researchers extend their appreciation to the Deanship of Scientific Research at King Saud University for funding the work through the research group project No. RGP-VPP-099.

\section{REFERENCES}

Abdel-Latif, A.S. and L.A.M. Abdallah, 2010. Two optimization methods to determine the rate constants of a complex chemical reaction using FORTRAN and MATLAB. Am. J. Applied Sci., 7: 509-517. DOI: 10.3844/ajassp.2010.509.517

Abd el-Salam, O.I., A.F.M. Fahmy, A.M. Mohamed, D.H. Elnaggar and A.G. Hammam, 2010. Synthesis, anticancer and anti-Inflammatory activities of 3, 4-dihydro-7- nitrobenzo[b]oxepin$5(2 \mathrm{H})$-one and its related derivatives. World $\mathrm{J}$. Chem., 5: 7-17. ISSN: 1817-3128.

Abou-Ghalia, M.H. and A.E. Amr, 2004. Synthesis and investigation of a new cyclo (Nalpha-dipicolinoyl) pentapeptide of a breast and CNS cytotoxic activity and an ionophoric specificity. Amino Acids, 26: 283-289. DOI: 10.1007/s00726-003-0042-8

Amr, A.E., A.M. Mohamed, S.F. Mohamed, N.A.A. Hafez and A.G. Hammam, 2006. Anticancer activities of some newly synthesized pyridine, pyraneand pyrimidine derivatives. Bioorg. Med. Chem., 14: 5481-5488. DOI: 10.1016/j.bmc.2006.04.045

Amr, A.E., K.A. Ali and M.M. Abdalla, 2009. Cytotoxic, antioxidant activities and structure activity relationship of some newly synthesized terpenoidal oxaliplatin analogs. Eur. J. Med. Chem., 44: 901-907. DOI: 10.1016/j.ejmech.2008.05.024

Atwal, K., 1988. Preparation of 2-amino dihydropyrimidin-5-carboxylates as cardiovascular agents. U.S. Patent, 4769371

Capdeville, R., E. Buchdunger, J. Zimmermann and A. Matter, 2002. Glivec (STI571, imatinib), a rationally developed, targeted anticancer drug. Nat. Rev. Drug Discov., 1: 493-502. PMID: 12120256

Chang, L.C., R.F. Spanjersberg, J.K. Frijtag, T.M. Krieger and G. denHout et al., 2004. 2, 4, 6Trisubstituted pyrimidines as a new class of selective adenosine A1 receptor antagonists. J. Med. Chem., 47: 6529-6540. PMID: 15588088

Chengguo, L.V., M. Lei and S. Yan, 2009. Study on the diurnal changes of net photosynthetic rate and the impact factors of Stevia rebaudiana bertoni in autumn. Am. J. Plant Physiol., 4: 18-23. 
Dahmardeh, M., 2011. Effect of plant density and nitrogen rate on PAR absorption and maize yield. Am. J. Plant Physiol., 6: 44-49.

Francis, B.J., B.J. Bency, P.K. Thorat and A.D. Lonkar, 2011. Gynandropsis pentaphylla DC extracts on the production of microbial proteins. Am. J. Drug Discovery Dev., 1: 129-136.

Fylaktakidou, C.K., J.D.L. Hadjipavlou, E.K. Litinas and N.D. Nicolaides, 2004. Natural and synthetic coumarin derivatives with anti-inflammatory/ antioxidant activities. Curr. Pharm. Des., 10: 38133833. PMID: 15579073

Gadhachanda, V.R., B. Wu, Z. Wang, K.L. Kuhen and J. Caldwell, et al., 2007. 4-Aminopyrimidines as novel HIV-1 inhibitors. Bioorg. Med. Chem. Lett., 17: 260-265. DOI: 10.1016/j.bmcl.2006.09.047

Hammam, A.G., A.F.M. Fahmy, A.E. Amr and A.M. Mohamed, 2003. Synthesis of novel tricyclic heterocyclic compounds as potential anticancer agents using chromanone and thiochromanone as synthons. Indian J. Chem. Sect. B., 42: 1985-1993.

Hammam, A.E.F.G., O.I.A. Salam, A.M. Mohamed and N.A.A. Hafez, 2005. Novel flouro substituted benzo[b]pyran with anti-lung cancer activity. ChemInform, 37: 1887-1893. DOI: 10.1002/chin.200602131

Hasanuzzaman, M., M.A. Hossain and M. Fujita, 2010. Physiological and biochemical mechanisms of nitric oxide induced abiotic stress tolerance in plants. Am. J. Plant Physiol., 5: 295-324. http://www.doaj.org/doaj?func=abstract \&id $=694001$

Henry, G.D., 2004. De novo synthesis of substituted pyridines, Tetrahedron, 60: 6043-6061. DOI: 10.1016/j.tet.2004.04.043

Ngoy, J.M., S.E. Iyuke, C.S. Yah and W.E. Neuse, 2011. Kinetic optimization of folic acid polymer conjugates for drug targeting. Am. J. Applied Sci., 8: 508-519. DOI: 10.3844/ajassp.2011.508.519

Jill, A.C., K.E. Atto, C.W. Howard and J.R. Schultz, 2011. Case report of severe injection site reaction associated with intramuscular naltrexone. Am. J. Pharmacol. Toxicol., 6: 1-4. DOI: 10.3844/ajptsp.2011.1.4

Joule, J.A. and K. Mills, 2000. Heterocyclic Chemistry. 4th Edn., Wiley-Blackwell, ISBN: 0632054530, pp: 589.

Jung, J.C., E.B. Watkins and M.A. Avery, 2005. Synthesis and cyclization reaction of pyrazolin-5one derivatives. Heterocycles, 65: 77-94.

Katritzky, A.R., C.W. Rees and E.F.V. Scriven, 1996. Comprehensive Heterocyclic Chemistry II: An Extended and Updated Review of the Literature
(1982-1995). 2nd Edn., Elsevier Science and Technology Books, ISBN: 0080420729, pp: 11628.

Khan, M.S.H., T. Wagatsuma, A. Akhter and K. Tawaraya, 2009. Sterol biosynthesis inhibition by paclobutrazol induces greater Aluminum (Al) sensitivity in Al-tolerant rice. Am. J. Plant Physiol., 4: 89-99. DOI: 10.3923/ajpp.2009.89.99

Li, A.H., S. Moro, N. Forsyth, N. Melman and X.D. Ji, 1999. Synthesis, CoMFA analysisand receptor docking of 3, 5-diacyl-2, 4-dialkylpyridine derivatives as selective A3 adenosine receptor antagonists. J. Med. Chem., 42: 706-721. PMID: 10052977

Lyle, G.G., J.J. Dziark, J. Connor and C. Saunderson, 1973. Structure of a dimeric piperidone-aldehyde condensation product. Tetrah, 29: 4039-4044. DOI: 10.1016/0040-4020(73)80234-0

Mallea, M., A. Mahamoud, J. Chevalier, S.A. Franco and P. Brouant et al., 2003. Alkylaminoquinolines inhibit the bacterial antibiotic efflux pump in multidrug-resistant clinical isolates. Biochem. J., 376: 801-805. PMID: 12959639

Matloobi, M. and C.O. Kappe, 2007. Microwaveassisted solution- and solid-phase synthesis of 2amino-4-arylpyrimidine derivatives. J. Comb. Chem., 9: 275-284. PMID: 17348733

Mcelvain, S.M. and K. Rorig, 1948. Piperidine derivatives: The condensation of aromatic aldehydes with 1-methyl-4-piperidone. J. Am. Chem. Soc., 70: 1820-1825. PMID: 18861789

Millet, J., M.T. Mdamet, S. Alibert, C. Rogier and C.S. Rouvier, et al., 2004. Dihydroethanoanthracene derivatives as in vitro malarial chloroquine resistance reversal agents. Antimicrob. Agents Chemother, 48: 2753-2756. PMID: 15215144

Naeem, M., M. Masroor A. Khan and J.B. Morris, 2009. Agrobotanical Attributes, nitrogen-fixation, enzyme activities and nutraceuticals of hyacinth bean (Lablab purpureus L.): A bio-functional medicinal legume. Am. J. Plant Physiol., 4: 58-69. DOI: 10.3923/ajpp.2009.58.69

Nasratun, M., H.A. Said, A. Noraziah and A.N.A. Alla, 2009. Immobilization of lipase from Candida rugosa on chitosan beads for transesterification reaction. Am. J. Applied Sci., 6: 1653-1657. DOI: 10.3844/ajassp.2009.1653. 1657

Negaoui, H., K. Hanane, K. Omar and S. Djamel, 2009. A model of intestinal anaphylaxis in whey sensitized Balb/c mice. Am. J. Immunol., 5: 56-60. DOI: 10.3844/ajisp.2009. 56.60 
Ozeki, K., T. Ichikawa, H. Takehara, K. Tanimura and M. Sato et al., 1989. Studies on antiallergy agents. III. Synthesis of 2-anilino-1, 6-dihydro-6-oxo-5pyrimidinecarboxylic acids and related compounds. Chem. Pharm. Bull., 37: 1780-1787. PMID: 2572333

Pattaraporn, C. and T. Vitidsant, 2009. Promotion of coconut shell gasification by steam reforming on nickel-dolomite. Am. J. Applied Sci., 6: 332-336. DOI: 10.3844/ajassp.2009. 332.336

Rezvani, S. and S. Shariati, 2010. Effect of trehalose Arabidopsis thaliana L. on huntington's disease. Am. J. Plant Physiol., 5: 1-6.
Shuangning, X., A. Shahbazi, L. Wang and C.W. Wallace, 2010. Supercritical ethanol liquefaction of swine manure for bio-oils production. Am. J. Eng. Applied Sci., 3: 494-500. DOI: 10.3844/ajeassp.2010.494.500

Vacher, B., B. Bonnaud, P. Funes, N. Jubault and W. Koek et al., 1999. Novel derivatives of 2-pyridinemethylamine as selective, potentand orally active agonists at 5-HT1A receptors. J. Med. Chem., 42: 1648-1660. PMID: 10229633

Velusamy, V. and L. Palaniappan, 2011. Compositional analysis $\alpha$-lactalbumin. Am. J. Biochem. Mol. Biol., 1: 106-120. 